\title{
Boundary Layer Turbulence over Surface Waves in a Strongly Forced Condition: LES and Observation
}

\author{
Nyla T. HusAin AND TETSu HARA \\ Graduate School of Oceanography, University of Rhode Island, Narragansett, Rhode Island \\ MARC P. BUCKLEY \\ Institute of Coastal Research, Helmholtz-Zentrum Geesthacht, Geesthacht, Germany \\ KiANOOSH Yousefi AND FABRICE VERON \\ College of Earth, Ocean, and Environment, University of Delaware, Newark, Delaware \\ Peter P. Sullivan \\ National Center for Atmospheric Research, Boulder, Colorado
}

(Manuscript received 19 March 2019, in final form 16 May 2019)

\begin{abstract}
The impact of sea state on air-sea momentum flux (or wind stress) is a poorly understood component of wind-wave interactions, particularly in high wind conditions. The wind stress and mean wind profile over the ocean are influenced by the characteristics of boundary layer turbulence over surface waves, which are strongly modulated by transient airflow separation events; however, the features controlling their occurrence and intensity are not well known. A large-eddy simulation (LES) for wind over a sinusoidal wave train is employed to reproduce laboratory observations of phase-averaged airflow over waves in strongly forced conditions. The LES and observation both use a wave-following coordinate system with a decomposition of wind velocity into mean, wave-coherent, and turbulent fluctuation components. The LES results of the mean wind profile and structure of wave-induced and turbulent stress components agree reasonably well with observations. Both LES and observation show enhanced turbulent stress and mean wind shear at the height of the wave crest, signifying the impact of intermittent airflow separation events. Disparities exist particularly near the crest, suggesting that airflow separation and sheltering are affected by the nonlinearity and unsteadiness of laboratory waves. Our results also suggest that the intensity of airflow separation is most sensitive to wave steepness and the surface roughness parameterization near the crest. These results clarify how the characteristics of finite-amplitude waves can control the airflow dynamics, which may substantially influence the mean wind profile, equivalent surface roughness, and drag coefficient.
\end{abstract}

\section{Introduction}

The coupling between wind and surface waves influences marine weather and climate by controlling the exchange of momentum, heat, and gases at the air-sea interface. Coupled ocean-atmosphere models have typically relied on a bulk parameterization of wind stress (or drag coefficient), which assumes a relationship with the neutral wind speed at $10 \mathrm{~m}$ above the sea surface. This simplification does not account for the effects of different

\footnotetext{
Corresponding author: Nyla Husain, nylahusain@uri.edu
}

sea states; in particular, it ignores intermittent and nonlinear wave-induced turbulent processes such as airflow separation and wave breaking. These have been shown to modify the local wind stress and surface roughness, causing deviations from the assumed logarithmic wind profile under varying wind-wave conditions (Banner and Melville 1976; Banner 1990).

Many previous modeling studies explored how different sea states (surface wave spectra) affect the drag coefficient (Makin and Kudryavtsev 1999; Hara and Belcher 2002, 2004), including some studies that explicitly accounted for the airflow separation effects (Kudryavtsev 
and Makin 2001; Kudryavtsev et al. 2001; Mueller and Veron 2009; Kukulka and Hara 2008). More recent studies considered the effects of complex sea states under tropical cyclones (Moon et al. 2003, 2004; Donelan et al. 2012; Reichl et al. 2014), including the impact of sea spray from breaking waves (Richter and Sullivan 2013; Veron 2015). However, uncertainty remains as to the observed relationship between the sea state and wind stress, especially under high wind conditions in which nonlinear turbulent processes dominate the airsea exchange (Black et al. 2007; Holthuijsen et al. 2012; Edson et al. 2013). It is apparent that these processes, which occur ubiquitously in the open ocean, produce cumulative effects on the drag coefficient and structure of the marine atmospheric boundary layer, but it is not clear how they manifest under different surface wave conditions. In fact, airflow separation has been viewed as a process which both limits form (pressure) drag in steep, nonbreaking wave conditions, and enhances the drag in breaking wave conditions (Donelan et al. 2004; Peirson and Garcia 2008; Grare et al. 2013). The contribution of the form drag to the total stress has been found to increase from incipient to active breaking waves (Banner 1990; Sullivan et al. 2018), but it is still unclear what role airflow separation plays in this transition.

Airflow separation has been well documented over breaking waves (Banner and Melville 1976; Reul et al. 2008) and has been observed intermittently over strongly forced nonbreaking waves using particle image velocimetry (PIV) in a wind-wave flume (Veron et al. 2007; Troitskaya et al. 2011; Buckley and Veron 2016), large-eddy simulation (LES; Sullivan et al. 2018), and direct numerical simulations (Yang and Shen 2010). It is characterized by a thin layer of enhanced shear and spanwise vorticity that detaches at the wave crest, generating turbulence and enhanced dissipation downstream. This separation often produces a low-velocity recirculation cell, or "bubble," on the lee side of the wave. Beyond these features, the airflow reattaches on the windward side of the following wave crest. Because the separation happens intermittently and fluctuates in size and intensity, the mean (phase-averaged) flow field does not show a clear flow separation pattern. Instead, the size of the recirculation cell of the mean flow following the phase speed increases as flow separation events become more frequent.

While the effect is seen in a range of sea states, the wave characteristics controlling its occurrence and intensity remain elusive. For example, it has been indicated that the wave steepness controls the magnitude of form drag (Peirson and Garcia 2008; Grare et al. 2013; Sullivan et al. 2018), but its effects on airflow separation processes that modulate the form drag are not well known. The surface roughness due to small-scale waves and breakers may also be connected to the occurrence of airflow separation over dominant waves and play a comparably important role in the form drag modulation (Gent and Taylor 1976; Donelan et al. 1993; Taylor and Yelland 2001; Kudryavtsev and Makin 2002).

In Hara and Sullivan (2015), an LES for wind over a sinusoidal wave train was employed to investigate how airflow dynamics are coupled to finite amplitude, strongly forced wind waves. The study revealed a signature of airflow separation characterized by enhanced turbulence above a recirculation cell on the lee side of the wave crest, accompanied by enhanced downward turbulent stress and mean wind shear at a similar elevation. It also confirmed the presence of large pressure form drag, reduction of turbulent stress and reduced mean wind shear closer to the surface. Variations in the mean wind profile due to turbulence in the airflow ultimately determine the equivalent surface roughness, or the drag coefficient modified by the surface waves.

In Buckley and Veron $(2017,2019)$, high-resolution PIV experiments enabled measurements of airflow dynamics very close to the surface under a range of windwave conditions. Instantaneous airflow separation patterns in observations resembled those exhibited by LES under similar conditions, showing promise that the airflow dynamics could be reproduced and their effects explored further in LES.

The present study seeks to determine to what extent LES can reproduce the observed airflow dynamics induced by strongly forced wind waves using horizontal mean and wave phase-averaged flow fields. By exploring the range of airflow patterns when wind-wave characteristics are altered, we aim to elucidate how finite amplitude surface waves modify the mean wind profile, equivalent surface roughness, and drag coefficient.

\section{Methods}

\section{a. Laboratory observation}

The measurements of airflow over wind waves used in this study are part of the extensive dataset acquired by Buckley (2015) (see also Buckley and Veron 2016, 2017, 2019). A full description of the experimental setup, methods, and results are described in Buckley and Veron (2017). These data were taken using a highresolution PIV system in a wind-wave flume measuring $42 \mathrm{~m}$ long, $1 \mathrm{~m}$ wide, and $1.25 \mathrm{~m}$ high with a water depth of $0.70 \mathrm{~m}$. The recirculating wind tunnel blew air directly over the water surface, and air was seeded with $O(10) \mu \mathrm{m}$ water droplets produced by a fog generator located at zero fetch. These droplets acted as Lagrangian tracers that moved with the airflow. 
The PIV setup was located at a fetch of $22.7 \mathrm{~m}$ where wind waves had sufficiently developed. The PIV system measured along-channel $2 \mathrm{D}$ velocity fields in the midsection of the flume by illuminating water particles with a high-intensity green laser sheet. By measuring the displacement of groups of water particles at a specified time interval, the instantaneous velocity fields were computed at a resolution of $O(100) \mu \mathrm{m}$. These velocity fields enabled the analysis of $2 \mathrm{D}$ wind vectors, turbulent kinetic energy (TKE), turbulent stresses, and vorticity.

Fluorescent dye was used in the water to detect the wavy surface on the same plane as the airflow measurements by using laser-induced fluorescence (LIF). The illumination of the water against a dark background enabled detection of the surface waves and measurement of their properties (i.e., wavelength, frequency, and amplitude). A separate camera with a large field of view was used to capture waves as they entered and exited the PIV field of view. These measurements were used to detect the wave phase along the surface by applying a Hilbert transform (Oppenheim and Schafer 2013; Buckley and Veron 2017). Laser wave gauges also measured time series of the wave height for each PIV image. Because laboratory wind waves are not strictly periodic, the wavenumber $k$ and the wave phase speed $c$ were determined from the observed peak frequency using the linear wave theory, and the wave amplitude $a$ was determined from the root-mean-square (RMS) wave amplitude.

In a wind-wave flume, wind is forced by a horizontal pressure gradient along the tank (i.e., pressure-driven channel flow). Far from the entry of the flume, where wind and wave fields vary slowly along the tank, this pressure gradient is approximately constant with height. The total wind stress (or flux) then varies linearly with height so that its vertical gradient is balanced by the horizontal pressure gradient (Uz et al. 2002; Zavadsky and Shemer 2012). This means that the so-called "constant flux" layer does not exist in the tank, and the stress actually decreases linearly toward the flume ceiling. The effect of this difference is expected to be small when focusing on wind fields very close to the water surface. For this study, the vertical wind stress profile was estimated using the PIV velocity measurements above the level of surface wave crests. The observed profile suggested that the stress magnitude decreased with height and approached zero toward the top of the flume due to relatively small friction at the ceiling.

We therefore fitted a linear stress profile to the observed profile, requiring that it reaches zero at the ceiling. This profile was extrapolated toward the surface to estimate the surface wind stress and the surface friction velocity $u_{*_{s}}$. We have ascertained that the results presented in this study are not sensitive to uncertainty of the stress profile in the tank, because our analyses focus on the wind field very close to the water surface.

In this study, comparison of observations with LES is performed using wave ages of $c / u_{s}=1.4$ and 1.6 (see Table 1). These conditions exemplified the two most strongly forced wind-wave fields recorded in the PIV experiments. At these wave ages, waves experienced occasional breaking, producing ripples of $O(1) \mathrm{cm}$ that visibly enhanced the surface roughness. Although airflow separation was also observed intermittently at higher wave ages $\left(c / u *_{s}=2.5\right.$ and 3.7), LES experienced limitations resolving the wind field over such waves. We believe that this limitation was caused by a reduction of larger-scale turbulence associated with a lower dominant (phase-averaged) wave slope and a lower value of background surface roughness (undeveloped small scale waves) in the laboratory, making LES more sensitive to unresolved (subgrid) motions and more difficult to obtain a stable solution. We expect that surface roughness in the open ocean is significantly more pronounced than in a laboratory flume at such wave ages, and LES is suitable for simulating airflow in such conditions. In fact, past studies have successfully used identical turbulence closure schemes to explore a broader range of wave ages and wave slopes with no model stability issues (see Sullivan et al. 2014, 2018). However, in a wind-wave flume such wave ages correspond to very low wind speeds with undeveloped higher-frequency waves, and the viscosity effects become important near the surface. It is possible that direct numerical simulation (DNS) would be more suitable for these more mature laboratory waves (i.e., Yang and Shen 2010) as they are able to resolve all scales of turbulence and viscous effects. Since our main goal is to validate LES results against laboratory observations, we focus on the two low-waveage conditions in this study.

It is important to note that the dominant laboratory waves of such wave ages are not common in the open ocean; that is, they are shorter and of higher frequency than typical waves near the spectral peak. However, investigating the airflow characteristics and the resulting enhancement of the drag coefficient over such waves is important, because it is well known that high-frequency waves represent an important part of the wave spectrum that can support a large portion of the air-sea momentum flux (Donelan et al. 2012; Reichl et al. 2014).

\section{b. Large-eddy simulation}

The LES methodology is employed using an approach identical to that of Sullivan et al. (2014), Hara and Sullivan (2015), and Sullivan et al. (2018). We define $t$ as time, $x$ as an along-wind coordinate, $y$ as a crosswind 
TABLE 1. Nondimensional parameters used in 11 LES experiments: wave age $c / u_{*_{s}}$, wave steepness $k a$, surface roughness $k z_{o}$, and surface drift velocity $u_{d} / u_{*_{s}}$. In the text and figure captions, surface roughness $k z_{o}=2.70 \times 10^{-3}, 1.35 \times 10^{-3}$, and $0.67 \times 10^{-3}$ are referred to as $k z_{o 1}, k z_{o 2}$, and $k z_{o 3}$, respectively. For run $3 \mathrm{c}$, $k z_{o 3 \text { new }}=k z_{o 3}$ from $k x=\pi / 2$ to $3 \pi / 2$.

\begin{tabular}{|c|c|c|c|c|}
\hline Run & $c / u *_{s}$ & $k a$ & $k z_{o}\left(\times 10^{-3}\right)$ & Perturbation \\
\hline 1 & 1.4 & 0.27 & 2.70 & - \\
\hline $2 \mathrm{a}$ & 1.4 & 0.27 & 1.35 & - \\
\hline $2 b$ & 1.4 & 0.20 & 1.35 & $k a_{\text {new }}=0.74 k a$ \\
\hline $3 \mathrm{a}$ & 1.4 & 0.27 & 0.67 & - \\
\hline $3 b$ & 1.4 & 0.27 & 0.67 & $\frac{u_{d}}{u *_{s}}=-\frac{a \omega}{u_{*_{s}}}[\cos (k x)-1]$ \\
\hline $3 \mathrm{c}$ & 1.4 & 0.27 & 0.67 & $\begin{array}{c}k z_{\text {o3new }}=k z_{o 3}[1+0.5 \cos (k x)] \\
\left(k x=\frac{3 \pi}{2} \text { to } \frac{\pi}{2}\right)\end{array}$ \\
\hline $3 d$ & 1.4 & 0.27 & 0.67 & $\frac{u_{d}}{u_{*}}=\frac{0.03}{u_{*}} U_{10}$ \\
\hline $3 \mathrm{e}$ & 1.4 & 0.27 & 0.67 & $\begin{aligned} u *_{s} & u_{*_{s}} \\
k z_{o 3 \text { new }}= & k z_{o 3}[1+0.5 \cos (k x)] \\
& (k x=0 \text { to } 2 \pi)\end{aligned}$ \\
\hline 4 & 1.6 & 0.26 & 2.70 & - \\
\hline 5 & 1.6 & 0.26 & 1.35 & - \\
\hline 6 & 1.6 & 0.26 & 0.67 & - \\
\hline
\end{tabular}

coordinate, and $z$ as a vertical upward coordinate with $z=0$ at the mean water surface, with velocities $(u, v, w)$ in $(x, y, z)$ directions. In this study we consider steady wind over a periodic surface wave train with the surface specified as $z=h(x, t)=a \cos (k x-\omega t)$, where $a$ is the wave amplitude, $k$ is the wavenumber, $\omega$ is the angular frequency, and $c=\omega / k$ is the phase speed. The phase speed $c$ and the wave orbital velocities at the water surface $(u, w)$ are specified based on the linear deep water wave theory, $c=\omega / k=\sqrt{g / k}, u=a \omega \cos (k x-\omega t)+u_{d}$, and $w=a \omega \sin (k x-\omega t)$, where $u_{d}$ is the surface drift velocity explained in the next section. Implications of using the sinusoidal wave shape and the linear wave phase and orbital velocities will be discussed later.

Turbulence in LES for wind over strongly forced waves is dominated by resolved large-scale eddies, and therefore less sensitive to subgrid-scale (SGS) motion. The turbulent flow in LES is thus spatially filtered, and the subgrid fluxes are parameterized using a conventional TKE-closure SGS parameterization described in detail in Moeng (1984), Sullivan et al. (2014), and Moeng and Sullivan (2015), and utilized by Hara and Sullivan (2015) and Sullivan et al. (2018). It models the SGS terms (including stress, energy flux, and energy dissipation) by relating SGS fluxes to the resolved-scale field through bulk transfer coefficients. This approach requires a roughness parameter to be imposed on the surface with a bulk aerodynamic formula for momentum (and scalars) applied point by point along the wavy boundary based on Monin-Obukhov (MO) similarity theory. The lower boundary is therefore parameterized with a surface roughness length $z_{o}$, which serves as a representation of the surface viscous stress effect and the roughness of smaller unresolved elements, such as higherfrequency waves. This roughness length relates the local instantaneous surface tangential stress and the tangential velocity at the first LES vertical grid level, with a log wind profile assumption in between. A free-slip (no tangential stress) condition is imposed at the upper boundary, where the surface is flat and vertical velocity disappears $(w=0)$.

Given the use of an SGS model in LES, the turbulent stress and TKE consist of both resolved and parameterized SGS contributions; the LES results presented in this study always combine the two. Because the high resolution [of $O(100) \mu \mathrm{m}$ ] PIV measurements resolve most of the turbulent eddy scales (Buckley and Veron 2017), it is reasonable to include the SGS contribution in LES results when comparing with PIV.

The mapping between physical vertical coordinate $z$ and the computational vertical coordinate $\zeta^{\mathrm{LES}}$ is given as

$$
z=\zeta^{\mathrm{LES}}+h(x, t)\left(1-\frac{\zeta^{\mathrm{LES}}}{l_{\zeta}}\right)^{3},
$$

so that the $\zeta^{\mathrm{LES}}$ coordinate follows the waves close to the surface, and gradually approaches the flat top boundary at $z=\zeta^{\mathrm{LES}}=l_{\zeta}$. The computational domain has a size of $l_{x} \times l_{y} \times l_{\zeta}$ where $l_{x}=l_{y}=5 \lambda$ and $l_{\zeta}=\lambda$, with $\lambda=2 \pi / k$ as the wavelength. It is discretized with $\left(N_{x}, N_{y}, N_{\zeta}\right)=(256,256,128)$ grid points, making the horizontal resolution $\Delta x=\Delta y=0.01953 \lambda$. Sullivan et al. (2018) considered a grid spacing identical to that of the present study as well as one with 4 times finer horizontal resolution, finding only small differences between the coarse and fine grid results. The vertical spacing ratio is nonuniform; the ratio between neighboring cells is held constant at 1.0028 , with the first point off the water surface located at $\zeta_{1}^{\mathrm{LES}}=0.0065 \lambda$. Waves propagate in and out of the computational domain in the positive $x$ direction with doubly periodic conditions imposed on the horizontal boundaries. Wind forcing is applied in the $x$ direction with a negative external pressure gradient $\partial P / \partial x$ that yields a negative surface stress, $\tau_{s}=(\partial P / \partial x) l_{\zeta}$, where $\tau_{s}$ is a sum of the form (pressure) stress and the viscous (subgrid) stress at the surface. The surface friction velocity is defined as $u_{s}=\left|\tau_{s}\right|^{1 / 2}$, where both $P$ and $\tau_{s}$ are already divided by air density so that they have a dimension of velocity squared. The simulation is run for approximately 60000 time steps and averaged over the last 20000 time steps after the wind field has become statistically steady. Sullivan et al. (2014) and Sullivan et al. (2018) provide a full description of the LES algorithm and numerical methods used to solve the governing equations. 


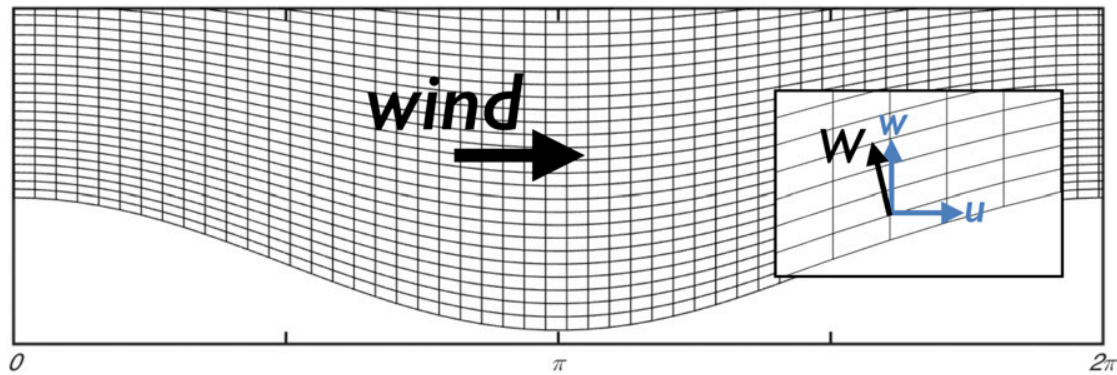

FIG. 1. Schematic of the wave-following mapping used for analysis of both LES and PIV results introduces a vertical coordinate $\zeta$ that follows the wave shape at $\zeta=0$ and gradually approaches the Cartesian $z$ coordinate as $\zeta$ increases. This mapping enables analyses below the wave crest level. The vertical momentum flux is defined using a Cartesian velocity $u$ and a contravariant velocity $W$ as shown in the small box.

\section{c. Wave-following mapping and triple decomposition}

The analyses of both LES and observation are performed in mapped wave-following coordinates (except for Fig. 2), and the turbulent and wave-induced perturbations in the airflow are analyzed by decomposing a given quantity into mean, wave-coherent, and turbulent fluctuation components, as in Hara and Sullivan (2015). Previous studies have also employed a similar wavefollowing coordinate system to study the partition of momentum flux in the wave boundary layer (Sullivan et al. 2000; Chalikov and Rainchik 2011).

With the LES results, we first introduce horizontal coordinates that move with the wave:

$$
\xi=x-c t, \quad \eta=y,
$$

so that the wave shape $h=a \cos (k \xi)$ becomes steady in time. The vertical coordinate is then mapped from $z$ to $\zeta$

$$
z=\zeta+a \cos (k \xi) e^{-k \zeta},
$$

with the Jacobian of the coordinate transformation

$$
J=\frac{\partial \zeta}{\partial z}
$$

so that $\zeta=0$ exactly follows the wave shape and $\zeta$ gradually approaches $z$ as it increases (see Fig. 1). This mapping enables us to examine the airflow everywhere in the field, including below the wave crest level. It differs from that of the LES computational domain in that the waviness of constant $\zeta$ lines decays exponentially, while the waviness of constant $\zeta^{\text {LES }}$ lines is forced to disappear at the fixed, flat top boundary. Therefore, the LES results are first mapped from the $\zeta^{\mathrm{LES}}$ coordinate to the $z$ coordinate, and then remapped to the $\zeta$ coordinate for analysis.
In this mapped coordinate system, each LES variable, $\psi$, is averaged in both $\eta$ and $t$, and is separated into a phase average $\bar{\psi}$ and a turbulent fluctuation $\psi^{\prime}$,

$$
\psi=\bar{\psi}+\psi^{\prime} .
$$

Next, the phase average $\bar{\psi}$, which is a function of $\xi$ and $\zeta$, is averaged in $\xi$ and is separated into a horizontal mean $\langle\psi\rangle$ and a wave-coherent $\tilde{\psi}$,

$$
\bar{\psi}=\langle\psi\rangle+\tilde{\psi},
$$

where $\langle\psi\rangle$ is a function of $\zeta$ only.

Hara and Sullivan (2015) provide a full description of this triple decomposition and derive the continuity, momentum, and energy equations in the mapped coordinate system. In particular, the $x$-momentum equation, normalized by the surface wind stress $\tau_{s}$, becomes

$$
\frac{\left\langle\tau^{w}\right\rangle+\left\langle\tau^{p}\right\rangle}{\tau_{s}}+\frac{\left\langle\tau^{t}\right\rangle}{\tau_{s}}+\frac{\frac{\partial P}{\partial x}}{\tau_{s}}=1 .
$$

Here, the horizontal mean wave-coherent stress $\left\langle\tau^{w}\right\rangle=$ $\langle\tilde{u} \tilde{W}\rangle$ and turbulent stress $\left\langle\tau^{t}\right\rangle=\left\langle u^{\prime} W^{\prime}\right\rangle$ are defined using a Cartesian velocity $u$ and a contravariant velocity

$$
W=\frac{1}{J} u \frac{\partial \zeta}{\partial x}+w
$$

which is perpendicular to a constant $\zeta$ surface. Therefore, the term $u W$ refers to flux of $x$ momentum across a constant $\zeta$ surface due to an advective velocity $W$ (see Fig. 1). The pressure stress is defined as

$$
\tau^{p}=\frac{1}{J} \bar{p} \frac{\partial \zeta}{\partial x}
$$

which also transfers $x$ momentum across the tilted constant $\zeta$ surface. Equation (7) shows that the total wind stress is a sum of the wave-coherent stress, pressure 
stress, and turbulent stress, varying linearly in the presence of the pressure gradient in the tank. In LES, the parameterized subgrid-scale viscous stress, which becomes significant toward the surface, is included within the turbulent stress.

The wave shape in the instantaneous observational flow fields is neither strictly periodic nor sinusoidal. First, the vertical coordinate is mapped from $z$ to $\zeta^{\prime}$ based on the observed instantaneous surface wave shape,

$$
z=\zeta^{\prime}+\sum_{n} a_{n} \cos \left(k_{n} x+\phi_{n}\right) e^{-k_{n} \zeta^{\prime}}
$$

where $a_{n}, k_{n}$, and $\phi_{n}$ are respectively the amplitude, wavenumber, and phase of the $n$th mode in the Fourier decomposition of the wave shape (Buckley and Veron 2016, 2017, 2019). Note that we have differentiated the PIV mapping $\left(\xi^{\prime}, \zeta^{\prime}\right)$ from that of $\operatorname{LES}(\xi, \zeta)$. This mapping is a generalized form of the mapping of (3) and differs from that of LES in that it accounts for multiple wave modes observed instantaneously while LES only contains one wave mode. Nevertheless, we have found that the phase-averaged wave shape from observations was very close to sinusoidal with no phase-locked higher-frequency wave modes visible. The phase of each observed wave is determined by applying a Hilbert transform directly to the observed wave profile, and the location of the left (right) crest of each wave is then assigned as $k \xi^{\prime}=0\left(k \xi^{\prime}=2 \pi\right)$. Note that the $k \xi^{\prime}$ value varies linearly with respect to the phase detected by the Hilbert transform and is not necessarily linear with respect to $x$ due to the asymmetry of instantaneous waves about the crest. Finally, the phase speed of waves at the observed peak frequency, based on linear wave theory, is subtracted from the observed horizontal velocity so that the phase-averaged quantities are approximately consistent with those of the LES results. More details on this phase averaging and its implications will be discussed in the results section.

\section{d. Matching experimental conditions}

All variables in LES are normalized by length scale $1 / k$ (inverse wavenumber), velocity scale $u_{*_{s}}$ (surface friction velocity), and time scale $1 / k u *_{s}$. Note that the surface wind stress (or the surface friction velocity squared $u_{*_{s}}^{2}$ ) is the surface value of the linearly varying total wind stress and it is a sum of the form stress and the subgrid (parameterized frictional) stress in LES. The key nondimensional parameters to be explored include the wave age $c / u *_{s}$, wave slope $k a$, the normalized surface drift velocity $u_{d} / u_{*_{s}}$, and the normalized surface roughness length parameter $k z_{o}$. The first two parameters are approximately matched between the LES and the observations (Table 1).
In observations, a fraction of the wind stress is supported by smaller waves (waves that are not used in the phase averaging) as well as by the surface viscous stress. In LES, these effects are accounted for by specifying the surface roughness parameterization $z_{o}$ (nondimensionalized by wavenumber $k$ ) which must be estimated empirically. Following Hara and Sullivan (2015), we start with a baseline surface roughness $k z_{o 1}=2.70 \times 10^{-3}$, and reduce it to a half and fourth of this value to align LES results more closely with observed wind speeds and to investigate the effect of the surface roughness parameterization on the dominant airflow $\left(k z_{o 2}=1.35 \times 10^{-3}\right.$ and $k z_{o 3}=0.67 \times 10^{-3}$, respectively; see Table 1). Therefore, a total of six baseline simulations (two wave ages based on observations, three surface roughness parameterizations) are performed (runs 1, 2a, 3a, 4, 5, and 6).

In addition to the baseline simulations, five perturbation simulations are performed with altered surface boundary conditions for the case of $c / u *_{s}=1.4$, including changes to the wave slope $k a$, surface drift velocity $u_{d} / u_{*_{s}}$, and along-wave surface roughness $k z_{o}$. The latter two surface boundary perturbations are separated into two simulations each: one with a perturbation to both the crest and trough, and one with a perturbation to the trough only.

By default, LES runs are conducted without a surface drift velocity. Therefore, we add $u_{d} / u_{*_{s}}$ to the surface boundary conditions for two simulations: one with a uniform along-wave drift velocity estimated to be $3 \%$ of the extrapolated $10-\mathrm{m}$ wind speed from observations (i.e., $u_{d} / u *_{s}=0.03 U_{10} / u *_{s}$, run $3 \mathrm{~d}$ ), and one variable (sinusoidal) along-wave drift velocity such that the drift increases toward the trough but disappears toward the crest \{i.e., $u_{d} / u_{*_{s}}=a \omega / u_{*_{s}}[1-\cos (k \xi)]$, run $3 \mathrm{~b}$ \}. The total horizontal surface velocity (sum of the drift and orbital velocities) becomes constant in this case.

Previous studies have shown that a variable surface roughness parameterization along the wave phase shifts the pressure phase and results in enhanced wave growth that aligns more closely with observations (Gent and Taylor 1976; Gent 1977; Kudryavtsev and Makin 2002). Therefore, we performed two simulations varying the surface roughness $k z_{o}$ along the wave: one in which we increase the roughness by $50 \%$ at the crest and reduce it by $50 \%$ at the trough relative to the constant $k z_{o 3}$ \{i.e., $k z_{o 3 n e w}=k z_{o 3}[1+0.5 \cos (k \xi)]$, run $3 \mathrm{e}$ \}, and one in which we reduce roughness by $50 \%$ at the trough only [i.e., $k z_{o 3 \text { new }}=k z_{o 3}[1+0.5 \cos (k \xi)]$ for $\pi / 2<k \xi<3 \pi / 2$, $k z_{o 3 n e w}=k z_{o 3}$ for $k \xi<\pi / 2$ and $3 \pi / 2<k \xi$, run $\left.3 \mathrm{c}\right\}$. These perturbation runs will illuminate the influence of uniform and along-wave variation of $k z_{o}$ and $u_{d} / u_{*_{s}}$ on the 

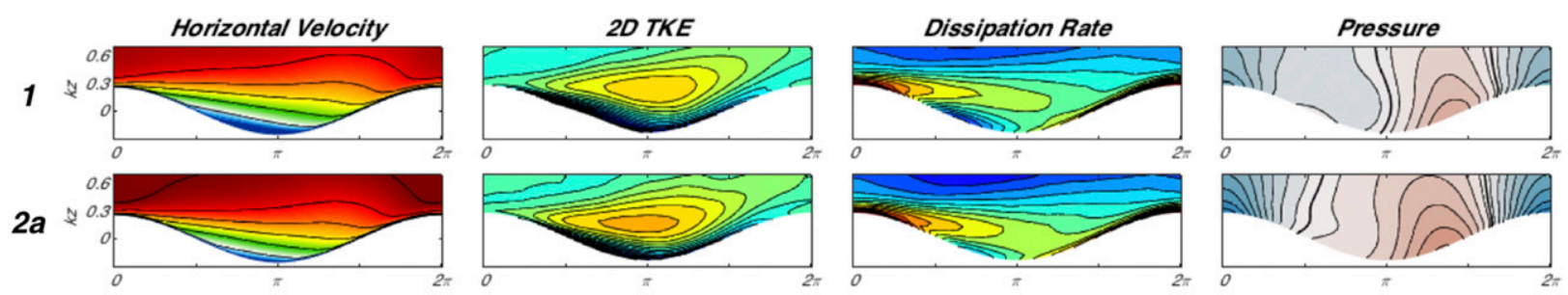

$2 b$
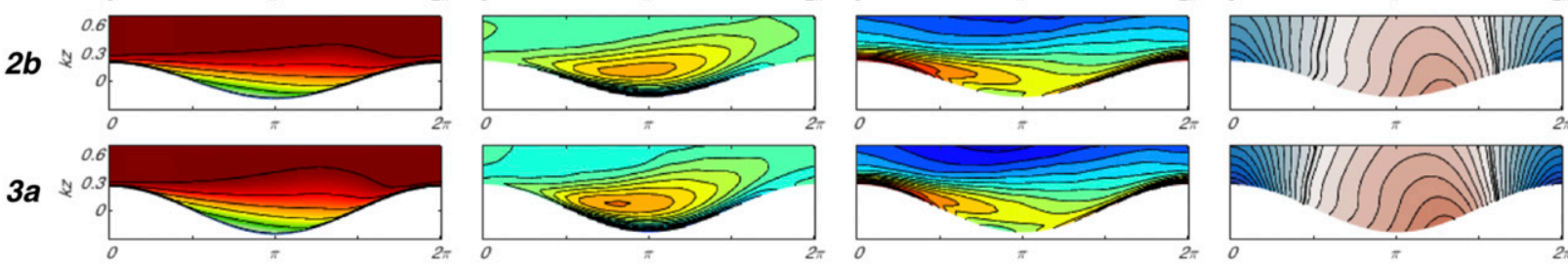

$3 b$
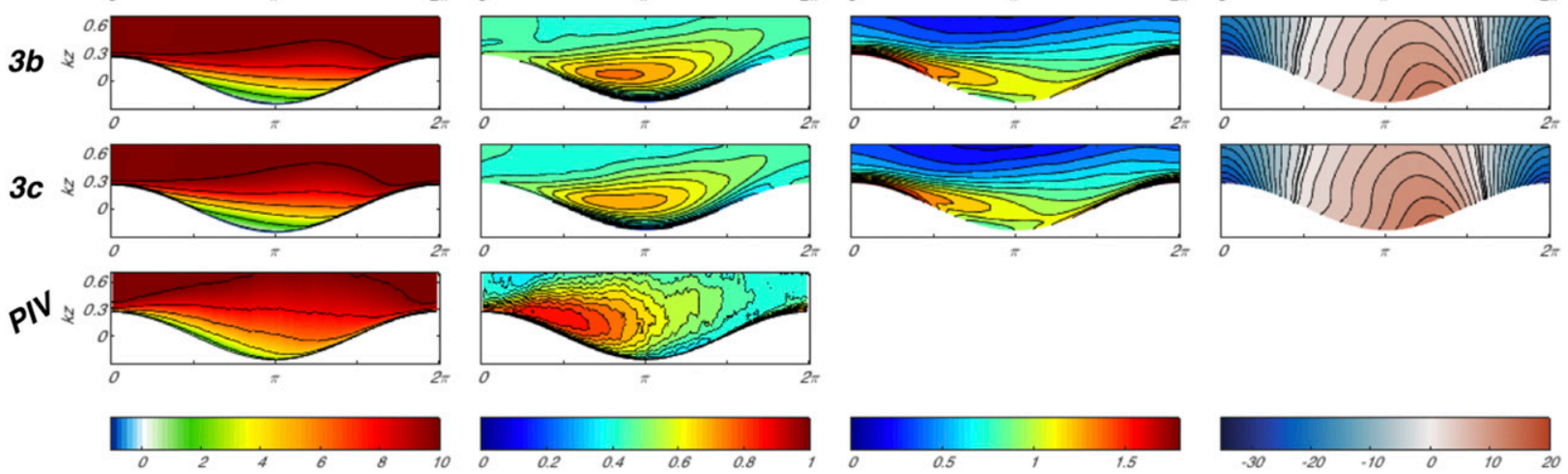

FIG. 2. Phase-averaged flow fields from left to right: horizontal velocity, two-dimensional turbulent kinetic energy, dissipation rate, and pressure. Each panel is labeled corresponding to its run number (see Table 1). Observations are shown in the bottom panel. All fields are nondimensionalized using surface friction velocity $u_{*_{s}}$, surface stress $\tau_{s}$, and wavenumber $k$.

structure of turbulence in the wavy boundary layer in LES.

Finally, another LES is conducted for the intermediate surface roughness $k z_{o 2}$ with a slightly reduced amplitude ( $74 \%$ of the baseline $\mathrm{ka}$ ) in order to investigate the response of airflow to modulations in wave steepness under strong wind forcing (run $2 b$ ).

\section{Results and discussion}

Note that for the remainder of our analysis, we directly compare the LES results in the $(\xi, \zeta)$ coordinates and the PIV observations in the $\left(\xi^{\prime}, \zeta^{\prime}\right)$ coordinates. We drop the primes from the definition of the PIV mapping.

\section{a. Results in physical coordinates}

In Fig. 2, phase-averaged LES and experimental results are presented over the physical wave following $\xi-z$ space for the case of $c / u *_{s}=1.4$. Note that the PIV is plotted as a function of $k \xi$ that is determined from phase detection using Hilbert transform. Snapshots of instantaneous waves appeared sharp-crested with flatter troughs, taking on a surface elevation asymmetry with respect to the mean water surface. While the elevation was asymmetrical, so too was the wave phase with respect to $x$; therefore, the phase-averaged wave shape in observations appeared very close to sinusoidal-that is, the phase detected by the Hilbert transform appeared to stretch/shrink the wave shape horizontally to make it more sinusoidal. Higher-frequency wave modes (ripples) were also observed instantaneously in the laboratory waves, but none were phase locked so as to appear in the phase-averaged wave shape. Thus, carrying out the LES and plotting the PIV results over a sinusoidal shape are both suitable for this comparison. The fact that the phase-averaging process using the Hilbert transform almost eliminates the asymmetry (nonlinearity) of the true observed wave shape is one potential reason for some of the discrepancy between the LES and the observation as discussed below.

Wind and waves are directed from left to right, and the phase speed $c$ has been subtracted from the horizontal velocities. Panels 1, 2a, and $3 \mathrm{a}$ (corresponding to the run names described above) in Fig. 2 show LES results for the three baseline surface roughness parameterizations in order of decreasing roughness $\left(k z_{o 1}, k z_{o 2}\right.$, and $k z_{o 3}$, 
respectively). Panel $2 \mathrm{~b}$ shows the results for $k z_{o 2}$ with wave steepness reduced to $74 \%$ of its baseline $(k a=0.20)$. Panel 3b shows the results for $k z_{03}$ with a variable surface drift velocity $u_{d} / u *_{s}$ that is zero at the crest and increases toward the trough. Panel $3 \mathrm{c}$ shows the results for a variable surface roughness that is equal to $k z_{o 3}$ near the crest and reduces by $50 \%$ at the trough. Panel PIV at the bottom shows corresponding observational results. The turbulent dissipation rate and pressure fields cannot be directly measured from these data, so they are not shown.

The leftmost column shows horizontal wind velocity. While it is not clearly visible in these flow fields, the wind velocity is always negative very close to the surface (moves from right to left) because the results are presented in a coordinate system moving at the wave phase speed; at the interface, the wind speed is equal to $-c$ plus the horizontal wave orbital velocity. A region of reduced horizontal velocity appears on the leeward side of the wave crest in all cases. When flow separates, it creates a large recirculation pattern (bubble) with a weak negative velocity in its lower half. As observed by Buckley and Veron (2016) and simulated by Sullivan et al. (2018), these reduced horizontal velocity patterns in the mean (phase-averaged) flow are associated with transient and intermittent airflow separation events that occur with different frequencies depending on the experimental conditions. No persistent separation events are observed in the instantaneous LES or PIV results.

In the LES with higher surface roughness (panels 1 and 2a), airflow separation events occur so frequently that even the mean flow shows a region of negative velocity. As the roughness is reduced in LES (from panels 1 to 2 a to $3 a$ ), the signature of airflow separation diminishes but does not disappear, leaving behind the appearance of sheltered but not fully separated flow. This indicates that instantaneous separation events become less frequent and less intense as surface roughness is reduced. The reduction of airflow separation due to reduced background surface roughness is qualitatively consistent with the observation by Gong et al. (1996) over a fixed sinusoidal topography.

Although they remain positive, the PIV velocities are dramatically reduced on the leeward side of the wave crest, making the general character and wind speed magnitude of the mean flow similar to LES particularly for the cases of $k z_{o 3}$ (panels 3a-3c). No obvious recirculation bubble is visible in PIV, but a sheltered region of reduced velocity can be observed on the leeward face of the crest, hugging close to the surface in a very thin layer upstream of the equivalent region in LES.

Although 3D TKE fields are available from LES results, PIV only measures the $x$ and $z$ components of velocity; thus, the two-dimensional TKE (2D TKE) is compared in the second column. The 2D TKE of LES includes two-thirds of the subgrid contribution, assuming isotropic turbulence in the subgrid scale. In both LES and PIV, the TKE is enhanced downstream of the crest away from the surface, and reduced in a thin layer close to the surface on its lee side. Enhanced TKE past the crest and away from the surface indicates advection of turbulent energy downstream by flow that has detached (or separated) from the surface. The advection of TKE may be influenced by the length of the detached free-shear layer before it loses coherence downstream. In PIV, the enhanced TKE begins and ends farther upstream and closer to the surface than LES, which suggests that turbulent energy is not advected as far downstream or away from the surface by the separated flow. These results reveal differences between LES and PIV in the behavior of airflow separation-particularly the character of shear layer detachment, sheltering, and reattachment of flow at the following crest. While reducing $k z_{o}$ in LES may improve the comparison of the mean wind speed, the character of phase-averaged shear layer detachment and sheltering still differs considerably from the laboratory observation.

The differences between LES and PIV could be caused by instantaneous changes to the location (phase) and angle of flow separation (detachment) and reattachment, both of which are observed to vary significantly from wave to wave in the tank. These variations may derive from a fluctuating, nonlinear laboratory wave shape that deviates from sinusoidal; an asymmetric wave shape can lead to asymmetry in wave orbital velocities, leading to a phase-dependent surface drift velocity. Additionally, at wave ages of $c / u *_{s}=2.5$ and younger, Buckley and Veron (2016) observed steepening and occasional breaking with air entrainment and bubble production. Roughness elements of $O(1) \mathrm{cm}$ (ripples) were visible extending above the viscous sublayer. These transient processes likely introduced modulations to the dominant wave slope $k a$, phase-dependent surface roughness $k z_{o}$, and surface drift velocity $u_{d} / u_{*_{s}}$. These effects are an expected consequence of a wind forcing regime in which very young waves transition from incipient to active breaking.

The existence of roughness elements and transient surface elevation modulations, especially at the crest, could explain the difference in the character of shear layer detachment observed in PIV. The contours of both TKE and horizontal velocity from PIV suggest that airflow may detach at a much steeper angle as compared to the LES runs with similar wind speed magnitude (panels 3a-3c). The airflow in PIV overshoots the height of the crest and produces a broad fan of reduced velocity 
downstream with little coherence. The cases of LES with higher surface roughness (panels 1 and 2a) also appear to detach at steeper angles that somewhat resemble PIV, but the downstream character of airflow is disparate. From these results, it appears LES is challenged to thoroughly reproduce the character of airflow in the observation using a steady, sinusoidal wave shape. Under this degree of wind forcing, it may be necessary to account for transient modulations to the spectral character of the waves, including phase-dependent parameters.

We suspected that the difference between the observation and the LES was partially caused by the enhanced/ reduced surface roughness (small scale waves) near the crest/trough of the observed waves, since the LES results with uniform along-wave surface roughness all underestimated the wind speed near the trough. We also suspected that the surface drift velocity might vary with phase and modify the flow field above. Thus, the effect of an along-wave variable surface roughness or an alongwave variable surface drift was addressed in a few perturbation runs for the case of $k z_{o 3}$ (runs $3 \mathrm{~b}, 3 \mathrm{c}$, and $3 \mathrm{e}$ ). From these runs, we have found that the flow field is mostly determined by the surface conditions (viz., the surface velocity and surface roughness length) near the crest, and that it is insensitive to the surface conditions near the trough. If the surface conditions are altered near the trough only (as in runs $3 b$ and $3 c$ ), the flow field hardly changes.

While we have anticipated the sensitivity of the flow to the crest conditions, the almost complete insensitivity to the trough conditions is surprising. To demonstrate the second point, we first show the case in which the roughness length is unchanged at the crest but reduced by $50 \%$ at the trough only (run 3c; Fig. 2, panel 3c). The result shows almost no difference from the baseline $k z_{o 3}$ case that shares the same roughness value at the crest but has double the roughness imposed at the trough. We have also performed another case in which the roughness is enhanced/reduced by $50 \%$ at the crest/trough (run $3 \mathrm{e}$, not shown), and have found that the flow field is not distinct from what we would expect if we imposed a $50 \%$ enhanced $k z_{o}$ that was uniform along the wave.

A similar pattern occurs with the addition of surface drift velocity. A surface drift added only at the trough (run 3b; Fig. 2, panel 3b) hardly affects the flow field at all compared to the baseline $k z_{o 3}$ case. A uniform (phase-independent) surface drift (run 3d; not shown) alters the flow field by simply increasing the phaseaveraged wind speed uniformly, but not affecting the wind shear or turbulence characteristics.

In summary, the surface boundary conditions near the crest (viz., horizontal surface velocity and surface roughness) appear to serve the foremost importance in the structure of airflow in LES, while conditions near the trough are of little importance. This finding also suggests that if the wave phase speed or orbital velocities are slightly modified (e.g., by accounting for the finite amplitude effect), the phase-averaged wind velocity will uniformly increase/decrease by the increase/decrease of total velocity at the crest (horizontal velocity + drift velocity - phase speed), but the other flow quantities will be hardly affected.

The turbulent dissipation rate in LES (Fig. 2, third column) shows enhanced dissipation of TKE at the trailing edge of the crest where wind shear is strongest. In all six LES cases shown, enhanced dissipation is advected downstream by the detached flow, but it is most apparent in the cases with higher surface roughness (panels 1 and $2 \mathrm{a}$ ). This is compatible with the advected 2D TKE shown in the second column. In the LES cases with reduced $k z_{o}$ and $k a$, the region of enhanced TKE and dissipation rate hugs closer to the surface, altering the airflow that presumably reattaches on the windward face of the following wave at a stagnation (or splat) point. There is a small region of reduced dissipation where similarly reduced TKE and horizontal velocity are present on the lee side of the wave, as observed by Hara and Sullivan (2015). This sheltered region (or dead zone) moves downstream and shrinks with reduced $k z_{o}$ and $k a$, most notably for the case with reduced $k a$. The splat point also changes with reduced $k z_{o}$ and $k a$, which is capable of modulating the pressure field over the wave (discussed next).

Pressure fields are shown in the fourth column of Fig. 2. The peak pressure is typically located on the windward face of the wave, near where flow reattaches at the splat point. As $k z_{o}$ is reduced, the splat point shifts upstream and the surface pressure is visibly enhanced. This effect is likely influenced by the character of flow separation that modifies the downstream airflow, including the force with which it strikes the surface. Strong separation upstream leads to turbulent flow carried farther downstream and dissipated, causing the splat to occur farther along the following wave with reduced force. This explains why lower $k z_{o}$ (which experiences less intense flow separation) produces a splat farther upstream toward the trough with more intense pressure.

The pressure fields for cases of $k z_{o 3}$ show more drastic along-wave pressure gradients. Reduced roughness at the crest allows the airflow to accelerate there, reducing the pressure and preventing strong flow separation or sheltering that reduces the mean wind speed. Again, these flow characteristics are primarily driven by surface boundary conditions near the crest where flow separation is initiated, and not at the trough. In the cases of 
enhanced surface drift velocity and reduced roughness at the trough only (panels $3 b$ and $3 c$, respectively), the nature of airflow separation in the physical flow fields does not visibly change from the baseline $k z_{o 3}$ (panel 3a). This again suggests that the sluggish airflow behind the wave during separation or sheltering diminishes the impact of the surface boundary conditions at that location.

Alterations to the pressure field by airflow separation modulate the phase-dependent surface pressure that determines the overall form drag. The case of reduced $k a$ begins to show distinctions from reduced $k z_{o}$ here, experiencing an upstream shift in the phase of peak pressure similar to the $k z_{o 3}$ but without the drastic along-wave pressure gradients. This result is a sign of a lower propensity for flow separation to occur over waves with a lower $k a$. The results for the case of reduced $k a$ are qualitatively consistent with the results of Donelan et al. (2006), who used in situ wave-following observations to analyze the correlation between wave slope and the occurrence of flow separation. They suggest a physical mechanism for flow separation whereby sharpening and steepening of the wave crest reduces the wave-induced vertical pressure gradient that maintains the centripetal acceleration necessary to keep streamlines attached to the surface. This causes flow to separate and ultimately the wave to spill over and break. While our LES did not experiment with the curvature at the wave crest, we altered the wave slope for run $2 b$ and found that its effects alone may play a significant role in modifying the frequency and strength of airflow separation.

\section{b. Results in mapped coordinates}

The wave-following mapping utilized in Hara and Sullivan (2015) is used to present the wind turbulence characteristics close to the surface. This mapping allows for an assessment of the effects of the wave shape at constant normalized heights above the waves $k \zeta$. Figure 3 shows the mean normalized horizontal wind speed minus the wave phase speed $(\langle u\rangle-c) / u_{*_{s}}$ for both wave ages (Fig. 3a), accompanied by the associated mean normalized wind shear (Fig. 3b) $(\partial\langle u\rangle / \partial \zeta)\left(\kappa \zeta / u *_{s}\right)$, and mean normalized 2D TKE (Fig. 3c) $\left(\left\langle u^{\prime} u^{\prime}\right\rangle+\right.$ $\left.\left\langle w^{\prime} w^{\prime}\right\rangle\right) / u_{* s}^{2}$.

Baseline LES runs are shown in dark blue with solid, dashed, and dotted lines characterizing $k z_{o}$ in order of decreasing roughness $\left(k z_{o 1}, k z_{o 2}\right.$, and $k z_{o 3}$, respectively). This line characterization is repeated for the perturbation runs using different colors: the dashed green line refers to $k z_{o 2}$ with reduced $k a$, and orange and purple dotted lines refer to the phase-dependent $u_{d} / u_{*_{s}}$ and $k z_{o 3}$ runs, respectively. PIV results are shown as solid black lines. The light gray line in column $b$ is the profile of the unperturbed wind shear experienced by the pressure-driven channel flow in both LES (solid) and PIV (dotted). As can be seen, the fitted wind shear profile in PIV is almost identical to LES.

All results are plotted in log scale in the vertical to focus on the effects very close to the surface. Below $k \zeta \approx 0.025$, results are omitted because the effects of unresolved viscosity and small-scale waves in LES become dominant. Above $k \zeta \approx 0.824$, PIV results are omitted due to noise. Above about $k \zeta=0.7$, the shape of the mean wind profile in LES returns to an almost straight line in log coordinates, indicating that at this location the wind approaches the typical logarithmic profile expected over a flat surface, slightly modified due to the slowly decreasing wind stress with increasing height above the water surface. Here, the LES differs from PIV in that the latter is still varying with height at the highest point of observation.

For both wind forcing cases, the magnitude of the mean wind in observations compares well with the cases of LES with $k z_{o 3}$. Below about $k \zeta=0.7$, the LES profiles deviate from the characteristic log profile for a flat surface from Monin-Obukhov similarity theory, indicating that the waves are altering the structure of the mean wind; this in turn will affect the equivalent roughness length $z_{o, e}$ (i.e., the parameter that sets the bulk surface roughness in the typical logarithmic wind profile). This wave-modulated deviation can be more readily seen in the profile of normalized mean wind shear (Fig. 3b), which shows regions of enhanced (reduced) wind shear to the right (left) of the unperturbed wind shear without waves (light gray line), respectively. The enhanced wind shear in this plot is indicative of the onset of airflow separation events that enhance the turbulence at a height comparable to the wave amplitude (see Fig. 2). Closer to the surface, wind shear is reduced to a value less than that expected over a flat surface. As Hara and Sullivan (2015) noted, the resulting $z_{o, e}$ is affected by the reduction of wind shear close to the surface, where momentum flux is dominated by wave-induced (pressure) stress. The magnitude and height of the enhanced wind shear are reduced as $k z_{o}$ is decreased, consistent with the physical LES velocity fields (Fig. 2). For the case with a reduced wave slope $k a$, the enhanced wind shear is shifted closer to the surface as expected, but does not reduce magnitude as it does for cases with $k z_{03}$; in fact, the wind shear is slightly enhanced above its baseline $k z_{02}$ in this case. Figure 2 sheds some light on the reason for this difference. The case of reduced $k a$ appears to separate somewhat differently than the cases of reduced $k z_{o 3}$; while streamlines stay tight to the surface in the former, the latter fans out more significantly downstream, producing slightly less TKE over the crest, but more above the trough (see Fig. 2, second 

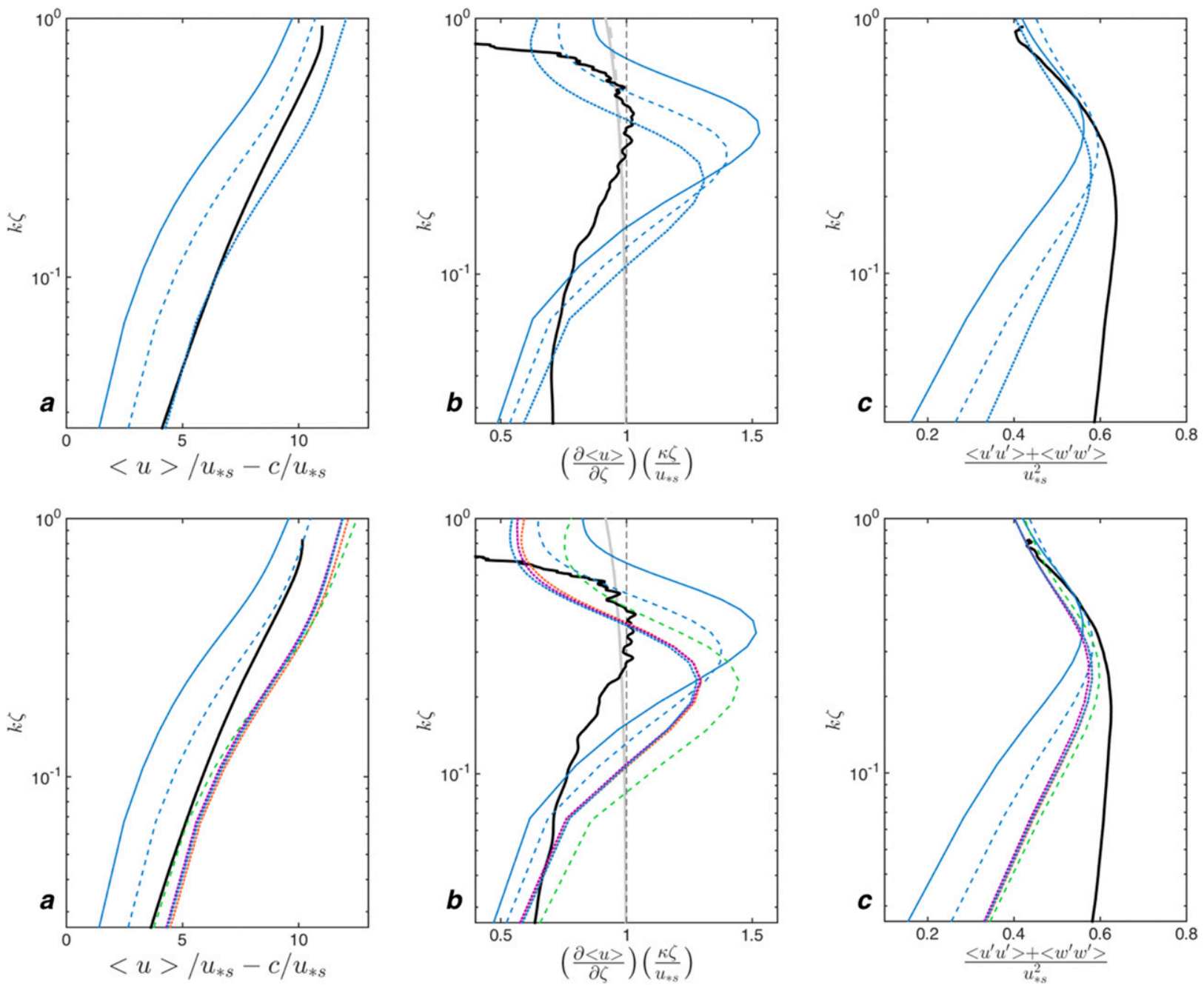

FIG. 3. Mean normalized (a) wind speed, (b) wind shear, and (c) 2D turbulent kinetic energy for (top) $c / u *_{s}=1.6$ and (bottom) $c / u_{*_{s}}=1.4$. Black lines show observational (PIV) results, while dark blue lines show LES results for three different surface roughness lengths: $k z_{o 1}$ (solid), $k z_{o 2}$ (dashed), and $k z_{o 3}$ (dotted). Perturbations include runs $2 \mathrm{~b}$ (green), $3 \mathrm{~b}$ (orange), and $3 \mathrm{c}$ (purple). All quantities are normalized using the surface friction velocity $u_{*}$. Gray lines (LES: solid; PIV: dashed) in the center panels refer to the unperturbed wind shear profile (without waves) accounting for the linear change in total wind stress with height. The results below $k \zeta \approx 0.025$ are omitted because the PIV profiles are strongly affected by viscosity and small-scale waves, and above $k \zeta \approx 0.824$ due to noisiness.

column). This effect is only slightly noticeable in the profiles of mean wind speed.

The PIV results also show enhanced wind shear near the crest level and reduced wind shear farther below. While wind shear from PIV is strongly reduced toward the surface as expected, enhanced wind shear is not nearly as prominent compared to LES. This effect is not entirely surprising considering the appearance of the physical flow fields (Fig. 2) suggest that the laboratory waves experience modulations to the wave parameters that impact the character of wave-produced turbulence. For example, the wave slope varies from wave to wave in the observations as waves intermittently break; this could enhance wind shear at different heights from wave to wave, smearing the mean wind shear profile. The LES case with reduced $k a$ exemplifies the potential effect wave slope modulations have on the wind shear. Nevertheless, the mildly enhanced wind shear from PIV is at a comparable height to LES, suggesting that the laboratory waves most often experience enhanced turbulence from instantaneous flow separation at this height. The mean 2D TKE (Fig. 3c) is enhanced at the same height for both LES and PIV, but the latter stays high closer to the surface. This is consistent with the results in Fig. 2. For all of our simulations, the subgrid contribution steadily increases toward the surface, but none reach a subgrid contribution over $60 \%$ of the total shown at the lowest point of the 2D TKE profile. Thus, 
we believe the differences between LES and PIV are not a result of the subgrid closure, but rather the wave perturbations in the tank not captured in the phaseaveraged wind fields.

\section{c. Momentum flux budget}

The momentum flux budget is derived from a triple decomposition of wind velocity into mean, turbulent, and wave-coherent components. This allows for a partition of the total momentum flux (or wind stress) into three contributions to describe how the waves alter the transfer of horizontal momentum across constant $\zeta$ surfaces: turbulent stress, wave-coherent stress, and pressure stress. The triple decomposition and derivation of the momentum and energy equations in wave-following coordinates for this study are described in more detail in Hara and Sullivan (2015), and have been used in past studies to describe the momentum flux partition in the presence of waves (Sullivan et al. 2000; Chalikov and Rainchik 2011). The turbulent (or Reynolds) stress $\tau^{t}$ is defined as the average of the product of turbulent fluctuation velocities $\left\langle u^{\prime} W^{\prime}\right\rangle$ and includes the subgrid-scale contribution. The wave-coherent stress $\tau^{w}$ is defined as the average of the product of wave-coherent velocities $\langle\tilde{u} \tilde{W}\rangle$, which arises when the phase-averaged wind velocity crosses the constant $\zeta$ surfaces. The pressure stress $\tau^{p}$, results from the pressure exerted on the tilted constant $\zeta$ surface, and is primarily responsible for wave growth at the surface.

Because wind is driven by a horizontal pressure gradient in LES and the laboratory flume, the total wind stress varies linearly and the full $x$-momentum equation includes the three stress contributions as well as the vertically integrated pressure gradient as in (7). The vertical profiles of the four terms on the left of (7) are plotted for LES and observations under both experimental wind-forcing conditions in Fig. $4\left(c / u_{*_{s}}=1.6\right.$ and 1.4 for the top and bottom panels, respectively). The results for wave-coherent stress, pressure stress, turbulent stress, and total wind stress including the pressure gradient are shown in Figs. 4a-d, respectively. The line colors and styles correspond identically with Fig. 3, except for the viscous stress from PIV (Fig. 4a; solid pink line). The pressure stress from PIV (Fig. 4b) is dot-dashed to signify that it has been calculated as the residual of the total wind stress after turbulent, wave-coherent, and viscous stress contributions have been removed; this is because pressure stress cannot be measured directly from the observations. The vertically integrated pressure gradient and the linearly decreasing wind stress which balances it - shown by gray lines for both LES (solid) and PIV (dashed) — are shown in Figs. 4b and 4c, respectively. Notice that both of these profiles turn out to be almost identical between the LES and the PIV for the case of $c / u *_{s}=1.4$.

It is important to note that sea spray has not been considered in this analysis, although it has been observed frequently over strongly forced conditions such as these, and has been suggested as a potential explanation for the reduction of the drag coefficient under tropical cyclone wind forcing (Powell et al. 2003; Donelan et al. 2004). Previous studies have assessed the impact of sea spray on air-sea momentum and energy fluxes (Fairall et al. 1994; Richter and Sullivan 2013; Veron 2015), and LES has proven to be a promising method for this type of investigation. Nevertheless, we have focused solely on the wave parameters in this study and will leave the effect of sea spray for a future study.

Both wind forcing cases $\left(c / u *_{s}=1.6\right.$ and 1.4) show similarities between LES and PIV in the horizontal mean partition of momentum flux (Fig. 4), especially in low roughness $\left(k z_{o 3}\right)$. Near the crest height, the mean normalized wave-coherent stress $\left(\left\langle\tau^{w}\right\rangle / \tau_{s}\right.$; Fig. 4a) is negative. Since each stress component is normalized by $\tau_{s}$ which is negative (indicating flux downward), a negative value of normalized wave-coherent stress is associated with upward momentum flux. This effect is the result of the wave-following coordinate system that sees accelerated mean flow that detaches (separates) away from the wavy surface toward a more positive constant$\zeta$ surface-that is, $\tilde{u}$ is positive (larger than the mean) and $\tilde{W}$ is positive (moving away from the surface) downwind of the peak of the wave crest, so the normalized wave-coherent stress is negative. When flow reattaches upwind of the following crest, $\tilde{u}$ is negative (smaller than the mean) and $\tilde{W}$ is also negative (moving toward the surface), so the normalized wave-coherent stress is again negative.

The spatial pattern of the phase-averaged wavecoherent stress can be seen in Fig. 5 (left column), where regions of negative normalized wave-coherent stress (upward flux) occur approximately at separation and reattachment points (from 0 to $\pi / 2$ and from $\pi$ to $3 \pi / 4$ ), and are much more intense than the positive (downward flux) regions where flow is more sluggish. This pattern is what causes a horizontal mean wave-coherent stress that is increasingly negative (upward flux) with enhanced airflow separation events and sheltering. In PIV, the wave-coherent stress is intensely negative at the approximate location of airflow separation but is not significant near the reattachment point, which supports our earlier suggestion that the laboratory waves experience some differences from LES in the character of flow separation and reattachment. For the case of the lowest roughness $\left(k z_{o 3}\right)$ in LES, wave-coherent stress is less intense at the point of separation, while for the case of 

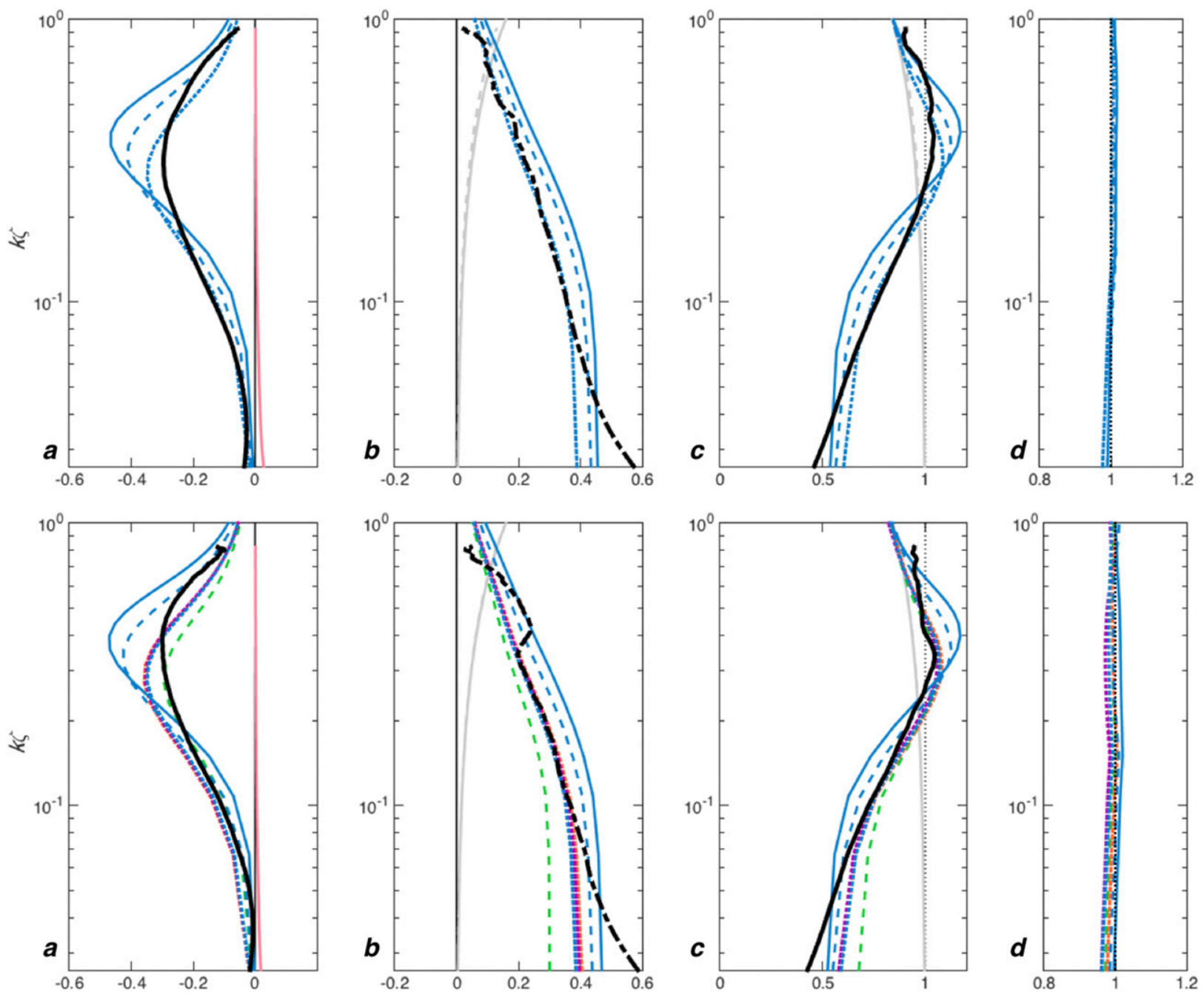

FIG. 4. Horizontally averaged profile of each normalized wind stress component for (top) $c / u *_{s}=1.6$ and (bottom) $c / u *_{s}=1.4$, with components shown as (a) wave-coherent stress $\left\langle\tau^{w}\right\rangle / \tau_{s}$ and viscous stress (pink, PIV only), (b) pressure stress $\left\langle\tau^{p}\right\rangle / \tau_{s}$ and vertically integrated pressure gradient $[(\partial P / \partial x) \zeta] / \tau_{s}$ (light gray), (c) turbulent stress $\left\langle\tau^{t}\right\rangle / \tau_{s}$ and linearly decreasing total wind stress $\tau_{\text {tot }} / \tau_{s}=1-(k \zeta / 2 \pi)$ (light gray), and (d) sum of all stresses including $[(\partial P / \partial x) \zeta] / \tau_{s}$ for each LES run, close to unity at all heights above the surface as expected. Black lines show observational (PIV) results, while dark blue lines show LES results for three different surface roughness lengths, with $k z_{o 1}$ (solid), $k z_{o 2}$ (dashed), and $k z_{o 3}$ (dotted). Perturbations include runs $2 \mathrm{~b}$ (green), $3 \mathrm{~b}$ (orange), and $3 \mathrm{c}$ (purple). The PIV pressure stress in (b) (dot-dashed) is estimated as the residual of all other stresses assuming linearly decreasing total wind stress. The results below $k \zeta \approx 0.025$ are omitted because the PIV profiles are strongly affected by viscosity and small-scale waves, and above $k \zeta \approx 0.824$ due to noisiness.

highest roughness $\left(k z_{o 1}\right)$ the stress at this location matches the magnitude of PIV more closely. Regardless, the wave-coherent stress in PIV averaged over the entire wave compares reasonably with the LES results, most closely with the $k z_{o 3}$ cases (Fig. 4).

Negative wave-coherent stress near the crest height is compensated by the enhancement of turbulent stress $\left\langle\tau^{t}\right\rangle / \tau_{s}$ (Fig. 4c) exceeding the total wind stress, which is correlated with enhanced mean wind shear near the peak height of the crest, as discussed by Hara and Sullivan (2015). The reduction of turbulent stress that can be seen close to the surface for both $c / u *_{s}=1.6$ and 1.4 is associated with increasing pressure stress, which is always positive for both LES and PIV for strongly forced waves (downward flux), as expected. This is correlated with the reduced mean wind shear toward the surface. For the case of $c / u *_{s}=1.4$ (Fig. 4 , bottom), the mean turbulent stress for the LES cases of $k z_{o 3}$ compare remarkably well with PIV above $k \zeta \approx 10^{-1}$. Below this level LES and PIV begin to diverge for both $c / u *_{s}=1.6$ and 1.4, with PIV experiencing a more rapid decrease in turbulent stress toward the surface. This divergence also 


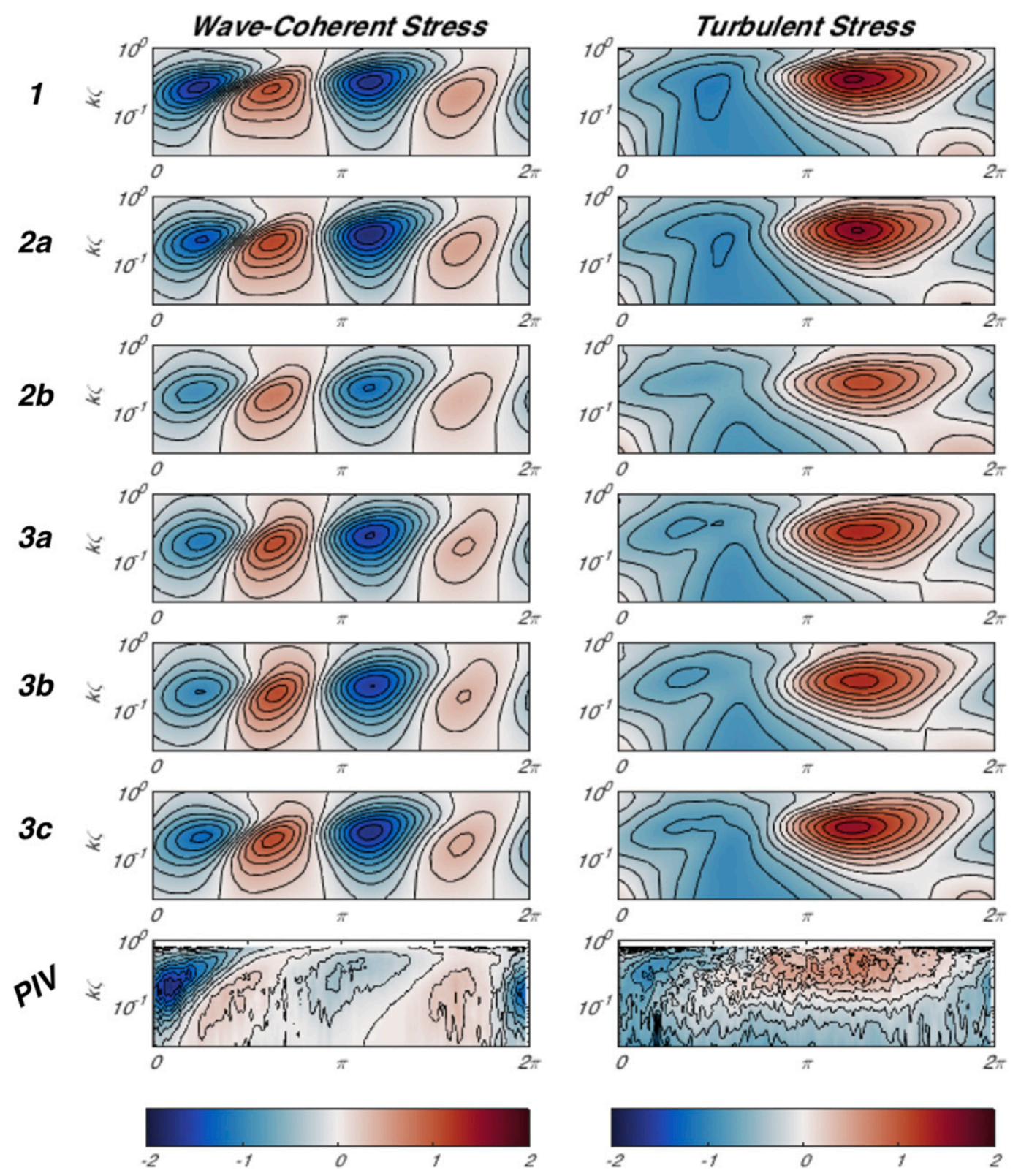

FIG. 5. Normalized phase-averaged mapped fields of wave-coherent stress and excess turbulent stress (turbulent stress minus total wind stress) for LES and PIV. Each panel is labeled corresponding to its run number (see Table 1). Observations are shown in the bottom panel. All results are normalized by the surface stress $\tau_{s}=u_{*_{s}}^{2}$.

causes diverging pressure stress contributions between the LES and PIV because the pressure stress is calculated as the residual of the total stress in PIV.

One possible reason for this divergence is that the LES turbulent stress is influenced by the SGS parameterization. In fact, below $k \zeta \approx 10^{-1}$, the SGS stress begins to dominate the total turbulent stress. However, we believe that the pressure stress component should remain relatively uniform with height near the wave surface because the pressure field itself is expected vary slowly with height (from the thin boundary layer approximation). It is more likely that the observed turbulent stress becomes less accurate very near the surface because of strongly fluctuating laboratory waves superimposed by smaller-scale waves.

\section{d. Surface stresses and wave growth rate}

Using the LES results, we next investigate how the surface stresses (pressure, normal turbulent stress, tangential turbulent stress) and the wave growth rate are 
modified by varying $k z_{o}, k a$, and $u_{d} / u_{*_{s}}$. The along-wave surface tangential stress is obtained by phase averaging the instantaneous surface tangential stress, which is calculated from the LES instantaneous tangential velocity at the first grid level and the specified roughness length $k z_{o}$. In reality, this tangential stress corresponds to a sum of the surface viscous stress and the form drag of smaller unresolved waves. In LES, the pressure and the normal stress are calculated at the first grid level, not at the surface. We have ascertained that a sum of the pressure and the normal stress (total normal stress) is approximately constant within the first few grid levels. Therefore, the true surface pressure is very close to the total normal stress evaluated at the first grid level. These phase-averaged surface stresses are shown in Fig. 6 for the wind forcing case of $c / u_{*_{s}}=1.4$.

The pressure fields in Fig. 2 and the pressure stress in Fig. 4 suggest that the surface pressure distribution is significantly modified by varying wave parameters, which is confirmed in the along-wave surface pressure distribution shown in Fig. 6. For $k z_{o 1}$, there is a region of very small negative and constant pressure below the dead zone produced by flow separation, roughly between $k x=\pi / 2$ and $\pi$. The peak surface pressure is significantly shifted downstream from the trough; that is, wind is pushing the wave. Reducing from $k z_{o 1}$ to $k z_{o 2}$ and then $k z_{o 3}$ weakens the flow separation effect (smoothing the surface pressure distribution below the dead zone), causing the phase of peak pressure to shift upstream slightly and the magnitude of the along-wave pressure gradient to increase significantly. As might be expected from our earlier results, the surface pressure distribution for the $k z_{o 3}$ perturbation runs in which surface boundary conditions are only altered in the trough barely change from their baseline $k z_{o 3}$. For the case of reduced $k a$ (dashed green line), the surface pressure distribution is "smoothed" similar to reducing $k z_{o}$, but the along-wave pressure gradient does not change drastically. This should be proportional to the wave amplitude according to the linear perturbation theory.

Using the phase-averaged surface stresses, the nondimensional wave growth rate coefficient $c_{\beta}$ is computed by finding the energy fluxes due to the (total) normal stress $\overline{\tau_{n}}$ and the tangential stress $\overline{\tau_{t}}\left(\mathrm{EF}_{n}\right.$ and $\mathrm{EF}_{t}$, respectively) into the waves as in Hara and Sullivan (2015):

$$
c_{\beta}=2 \frac{\mathrm{EF}}{u_{*_{s}}^{3}} \frac{u_{*_{s}}}{c}(k a)^{-2},
$$

where
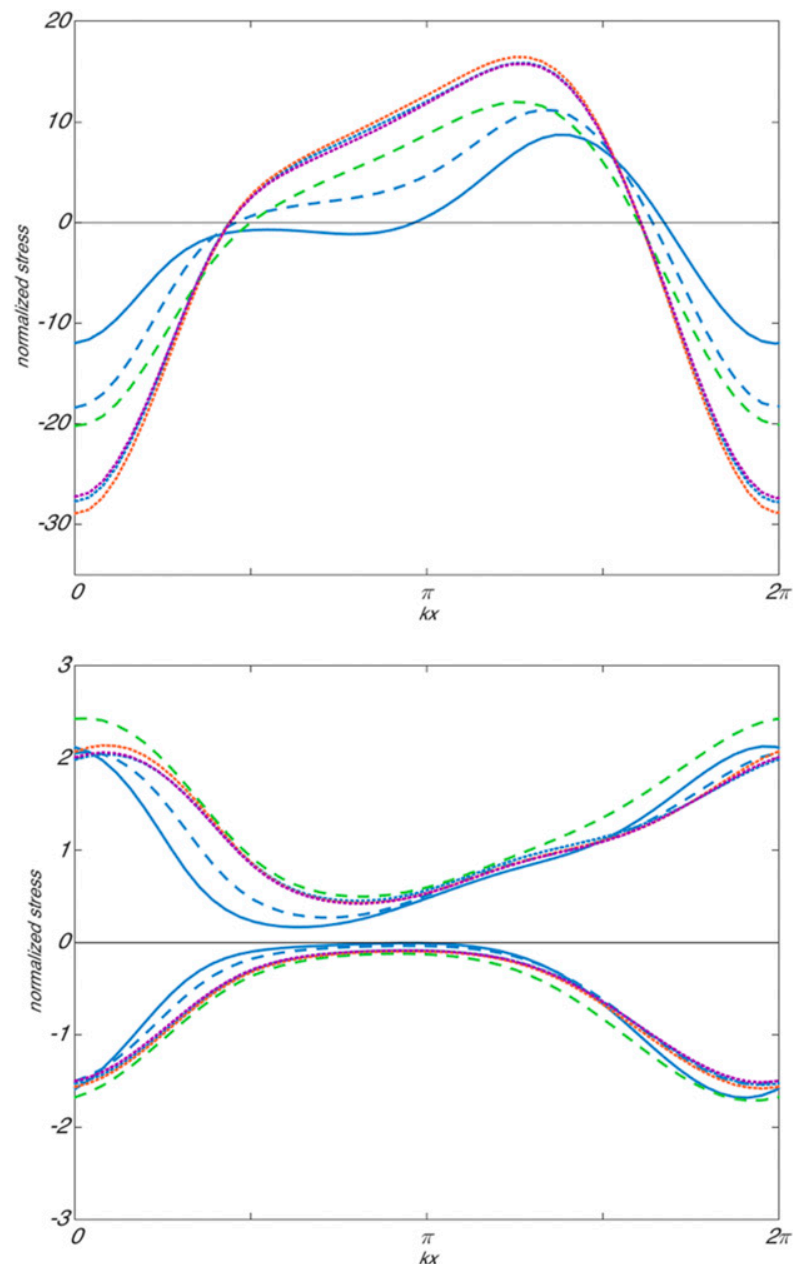

FIG. 6. Surface stress components for $c / u_{*_{s}}=1.4$, with (top) pressure contribution and (bottom) turbulent normal stress (above zero) and turbulent tangential stress (below zero). Dark blue lines show LES results for three different surface roughness lengths, with $k z_{o 1}$ (solid), $k z_{o 2}$ (dashed), and $k z_{o 3}$ (dotted). Perturbations include runs $2 \mathrm{~b}$ (green), $3 \mathrm{~b}$ (orange), and $3 \mathrm{c}$ (purple). All results are normalized by the total wind stress or surface friction velocity squared $\tau^{\text {wind }}=u_{*_{s}}^{2}$.

$$
\begin{aligned}
\mathrm{EF}_{n} & =\left\langle\frac{\overline{u_{n}} \overline{\tau_{n}}}{\cos \theta}\right\rangle, \quad \mathrm{EF}_{t}=\left\langle\frac{\overline{u_{t}} \overline{\tau_{t}}}{\cos \theta}\right\rangle \quad \text { and }, \\
\mathrm{EF} & =\mathrm{EF}_{n}+\mathrm{EF}_{t},
\end{aligned}
$$

where $\theta$ is the point-by-point angle of surface tilt from the horizontal, and $\overline{u_{n}}$ and $\overline{u_{t}}$ are normal and tangential components of the wave orbital velocity at the surface, respectively.

The total wave growth coefficient $c_{\beta \text { tot }}$ for each LES condition is listed in Table 2, along with its tangential stress, normal stress, and pressure stress contributions $\left(c_{\beta t}, c_{\beta n}\right.$, and $\left.c_{\beta p}\right)$. As expected, the wave growth is dominated by the top and bottom and lower panels of 
TABLE 2. Values of nondimensional wave growth coefficient $c_{\beta}$ for both wind forcing cases $\left(c / u_{s}=1.4\right.$, top; $c / u_{*_{s}}=1.6$, bottom). The values for $c_{\beta \text { tot }}, c_{\beta t}, c_{\beta n}$, and $c_{\beta p}$ refer to total, tangential stress, normal stress, and pressure contributions of $c_{\beta}$. For references to runs, refer to Table 1 .

\begin{tabular}{lrrrrrrrr}
\hline \hline Run & \multicolumn{1}{c}{1} & \multicolumn{1}{c}{$2 \mathrm{a}$} & \multicolumn{1}{c}{$2 \mathrm{~b}$} & \multicolumn{1}{c}{$3 \mathrm{a}$} & \multicolumn{1}{c}{$3 \mathrm{~b}$} & $3 \mathrm{c}$ & $3 \mathrm{~d}$ & $3 \mathrm{e}$ \\
\hline$c_{\beta \text { tot }}$ & 17.5 & 15.9 & 20.1 & 14.1 & 14.6 & 14.4 & 14.1 & 15.4 \\
$c_{\beta t}$ & 2.7 & 2.6 & 3.8 & 2.6 & 2.7 & 2.6 & 3.2 & 2.2 \\
$c_{\beta n}$ & 1.6 & 1.0 & 0.9 & 0.3 & 0.2 & 0.3 & 0.1 & 0.5 \\
$c_{\beta p}$ & 13.2 & 12.4 & 15.4 & 11.2 & 11.7 & 11.5 & 10.8 & 12.8 \\
Run & \multicolumn{1}{c}{4} & \multicolumn{1}{c}{5} & & 6 & & & & \\
\hline$c_{\beta \text { tot }}$ & 18.3 & 16.9 & - & 14.9 & - & - & - & - \\
$c_{\beta t}$ & 2.8 & 2.7 & - & 2.6 & - & - & - & - \\
$c_{\beta n}$ & 1.7 & 1.1 & - & 0.4 & - & - & - & - \\
$c_{\beta p}$ & 13.9 & 13.2 & - & 11.8 & - & - & - & - \\
\hline
\end{tabular}

Fig. 6. As $k z_{o}$ is reduced, the total wave growth rate mildly decreases. Although the along-wave pressure gradient increases with reduced $k z_{o}$, the phase of the peak pressure shifts toward the trough and reduces the form drag (Fig. 6). The addition of $u_{d} / u_{*_{s}}$ for runs $3 \mathrm{~b}$ and $3 \mathrm{~d}$ has almost no impact on the total growth rate. The phase-dependent $k z_{o}$ varying from baseline in the trough only (run $3 \mathrm{c}$ ) also has little impact on growth rate; that varying from baseline at both the crest and trough (run $3 \mathrm{e}$ ) approaches the $2 \mathrm{a}$ case where the roughness near the crest is the same. The most interesting finding is that the total growth rate significantly increases (by $26 \%$ ) as the wave slope $k a$ decreases (by $26 \%$ ). This is qualitatively consistent with the earlier finding that the pressure variation does not scale with the wave steepness. This means that the concept of the linear wave growth rate (the wave growth rate being independent of wave slope) does not apply here, which is consistent with the observational results of Peirson and Garcia (2008).

Between the two wind forcing conditions (top and bottom in Table 2), the higher wave age $\left(c / u_{s}=1.6\right.$, bottom) has a slightly higher growth rate (by $5 \%-6 \%$ ), primarily caused by higher-pressure contribution (turbulent normal and tangential contributions change very little). The value of $k a$ for this condition is slightly lower (by $4 \%$ ). It is therefore possible that this increase of the growth rate is mainly due to the associated decrease of $k a$ rather than the change in the wave age.

Many operational wave models assume that the wave growth rate is independent of wave slope and is related to the wind stress or the wind speed for a given wavenumber. Our results, however, show that the growth rate is dependent on the wave slope, consistent with the findings of Sullivan et al. (2018). When the surface roughness (effect of unresolved small waves) varies, the growth rate also changes even though the wind stress is held constant; that is, the growth rate is not determined by
TABLE 3. Values of normalized equivalent surface roughness $k z_{o e}$ for both wind forcing cases $\left(c / u *_{s}=1.4\right.$, top; $c / u *_{s}=1.6$, bottom). The value of $z_{o, e} / z_{o}$ is the ratio of the equivalent surface roughness $z_{o, e}$ to the parameterized surface roughness $z_{o}$. For references to runs, refer to Table 1.

\begin{tabular}{lcccccccc}
\hline \multicolumn{1}{c}{ Run } & 1 & $2 \mathrm{a}$ & $2 \mathrm{~b}$ & $3 \mathrm{a}$ & $3 \mathrm{~b}$ & $3 \mathrm{c}$ & $3 \mathrm{~d}$ & $3 \mathrm{e}$ \\
\hline$k z_{o, e} \times 10^{-3}$ & 11.51 & 7.90 & 3.55 & 4.53 & 4.10 & 4.57 & 4.41 & 5.47 \\
$z_{o, e} / z_{o}$ & 4.26 & 5.85 & 2.63 & 6.77 & 6.12 & 6.82 & 6.59 & 8.16 \\
$\quad$ Run & 4 & 5 & & 6 & & & & \\
\hline$k z_{o, e} \times 10^{-3}$ & 10.74 & 7.24 & - & 4.24 & - & - & - & - \\
$z_{o, e} / z_{o}$ & 3.98 & 5.36 & - & 6.32 & - & - & - & - \\
\hline
\end{tabular}

the wind stress alone. As the surface roughness decreases, the wind speed increases, but the growth rate decreases. Therefore, the growth rate is not determined by the wind speed alone, but rather is modified by both the wave slope and the surface roughness.

\section{e. Wave effect on equivalent surface roughness}

Since the mean wind speed profiles of LES roughly approach those over a flat surface (logarithmic profiles slightly modified by the slowly decreasing wind stress with height) for $k \zeta>0.7$ (Fig. 3, left), the equivalent surface roughness $z_{o, e}$ can be estimated by extrapolating the profiles above $k \zeta>0.7$ toward the surface. The results of $k z_{o, e}$ as well as the ratio $z_{o, e} / z_{o}$ (the enhancement of roughness length by the waves) are summarized in Table 3 . Note that the quantity $\log \left(z_{o, e} / z_{o}\right)$ is proportional to the increase of wind speed outside the wave boundary layer due to the wave. Hara and Sullivan (2015) discuss how $z_{o, e} / z_{o}$ increases if the normalized mean wind shear is reduced near the water surface, and decreases if the normalized mean wind shear is enhanced due to flow separation, as shown in Fig. 3 (center). The total wave effect on $z_{o, e}$ and the drag coefficient is determined by a sum of these two effects.

As the specified normalized surface roughness $k z_{o}$ decreases, $k z_{o, e}$ naturally decreases as well, but much less so; thus, the ratio $z_{o, e} / z_{o}$ increases significantly. Therefore, the wave effect on increasing surface roughness is more pronounced as the surface roughness decreases. This is consistent with the fact that decreasing surface roughness weakens the flow separation effects and the associated enhancement of mean wind shear. It is interesting that a similar effect was seen in a recent modeling study for turbulence in the oceanic wave boundary layer (Teixeira 2018); the equivalent (or effective) surface roughness was obtained by extrapolating the logarithmic current profile and showed enhancement over the imposed value in conditions of wave-dominated turbulence (see their Fig. 3).

As the wave slope $k a$ decreases, $z_{o, e} / z_{o}$ significantly decreases as expected. Adding constant surface drift 
velocity $u_{d} / u_{*_{s}}$ seems to shift the wind speed almost uniformly, inside and outside the wave boundary layer, but does not significantly affect $k z_{o, e}$, since this value is found after subtracting $u_{d} / u_{*_{s}}$ from the mean wind speed profile. Since adding a phase-varying $u_{d} / u_{*}$ that approaches zero toward the crest does not visibly affect the wind speed (as in run $3 b$ ), it appears that the presence of $u_{d} / u *_{s}$ at the crest dominates the airflow characteristics, a similar effect to when $k z_{o}$ is imposed at the crest. When constant surface drift is imposed along the wave (run 3d) the turbulent features in the airflow remain intact, and the only visible difference from the baseline is a small, vertically uniform increase in the wind speed. This suggests that the wave effect on the turbulence is not significantly altered by the surface drift.

\section{Summary}

Wind turbulence over strongly forced wind waves was simulated using LES and was compared with laboratory PIV observations for wave ages of $c / u *_{s}=1.4$ and 1.6 (very young waves) and respective wave slopes of $k a=0.27$ and $k a=0.26$. LES was performed over a periodic sinusoidal wave train and the results were phase averaged. Since the laboratory waves were solely wind generated, they were not strictly periodic, and hence the Hilbert transform of the wave elevation record was used to determine the wave phase before phase averaging of the results. Although the laboratory waves experienced frequent modulations to the wave slope, wave shape, and visible surface roughness, the phaseaveraged laboratory wave shape appeared remarkably sinusoidal. Therefore, we have used a sinusoidal wave shape for the phase-averaged LES comparison. Three different values of constant surface roughness (representing the effect of the surface viscous stress and the form drag of unresolved small waves) were used to match the wind speed in LES with the observation $\left(k z_{o 1}, k z_{o 2}\right.$, and $\left.k z_{o 3}\right)$.

The results show a reasonable comparison of LES and PIV, especially for the case of $k z_{o 3}$ conditions in LES. Both results show the signature of instantaneous airflow separation events (or sheltering), enhancement of the horizontally averaged wind shear profile near the crest, and reduction toward the surface (Fig. 3). They also show similar patterns of wave-coherent and turbulent stress which result in a good agreement of the momentum flux budget, particularly for the LES case of $k z_{o 3}$ (Figs. 4 and 5). However, important differences are observed in the mean flow patterns and the spatial distribution of 2D TKE, particularly near the crest and downstream. The disparity in the magnitude and location of turbulent features may have resulted from a number of differences between LES and the laboratory setup. These include the spectral spread, transient modulations, smaller-scale waves, and nonlinearity of the wind-generated laboratory waves compared with the periodic monochromatic waves modeled in LES, all of which can influence phase determination and averaging. The results suggest that wave characteristics at the crest play a key role in determining how the flow detaches/ reattaches on the leeward/windward face of the wave when it is strongly forced, as has been found by previous studies such as Donelan et al. (2006) mentioned earlier. Airflow over laboratory waves appears to detach at a steeper angle than LES while at the same time hugging closer to the surface and showing a somewhat incoherent detached free-shear layer. LES exhibits a more coherent signature of airflow separation, suggesting that instantaneous airflow separation events are more consistently advecting turbulence away from the surface.

To address these disparities, five LES perturbation runs were performed: one with a $26 \%$ reduced wave slope $k a$ (run $2 \mathrm{~b}$ ), two with constant and phase-varying surface drift velocity (runs $3 \mathrm{~d}$ and $3 \mathrm{~b}$ ), and two with phase-varying surface roughness (runs $3 \mathrm{c}$ and $3 \mathrm{e}$ ). Airflow in the runs with phase-varying surface boundary conditions showed no sensitivity to modifications near the trough, indicating that the conditions near the crest play a primary role in the turbulent flow over strongly forced waves. The LES results also show strong sensitivity to the wave slope $k a$ while maintaining the same wind forcing. Decreasing $k a$ lowers the height of enhanced mean wind shear and the signature of airflow separation events in general, which could explain disparities between LES and PIV if laboratory waves are experiencing frequent amplitude modulations. While decreasing $k z_{o}$ mildly increases the equivalent roughness length $\left(z_{o e} / z_{o}\right)$ and decreases the wave growth rate, decreasing $k a$ reduces $z_{o e} / z_{o}$ and significantly increases the wave growth rate (Tables 2 and 3), in agreement with Peirson and Garcia (2008) and Sullivan et al. (2018).

Overall, the results presented here demonstrate reasonable fidelity of LES to represent wind over surface waves in strongly forced conditions in a wind wave flume, particularly for horizontally averaged quantities, and illuminate the effect of surface waves on the mean wind profile and equivalent surface roughness (drag coefficient). Namely, near the height of the wave amplitude, transient flow separation causes upward wavecoherent stress, enhanced downward turbulent stress, and enhanced mean wind shear. Farther below, the downward pressure stress is balanced by the reduced downward turbulent stress and reduces the mean wind shear. 
These findings are consistent with those by Hara and Sullivan (2015), and can be used to develop improved parameterizations of sea-state-dependent air-sea momentum flux (drag coefficient).

The results also raise questions as to the surface boundary conditions needed to adequately represent wave fluctuations and spectral spread in a wind-wave regime of this nature. The present results suggest that the effects of wave characteristics specifically near the crest should be explored further. More work is required to understand the disparities in the phase-averaged character of instantaneous airflow separation events under strongly forced conditions, including the location, intensity, and angle of detachment and reattachment of airflow at the surface. It would also be desirable to use LES to further explore the effects of sea spray (Richter and Sullivan 2013), unsteady waves (Sullivan et al. 2018), and fully coupled wind and waves (Chalikov and Rainchik 2011) in gaining a more thorough understanding of a strongly forced wind-wave regime on airsea momentum flux and wave growth rate. Additionally, we would like to extend the present LES study to explore airflow characteristics of conditions that are difficult to achieve in laboratory observations, including misaligned wind-wave conditions, more mature wave ages, and the effects of surface waves on mean temperature, humidity, and scalars, including their transfer coefficients.

Acknowledgments. We acknowledge support of the National Science Foundation (Physical Oceanography) Grants OCE-1458984 (URI), OCE-1458977 (UD), and OCE-1634051 (UD). We also acknowledge highperformance computing support from Yellowstone (ark:/85065/d7wd3xhc) and Cheyenne (doi:10.5065/ D6RX99HX) provided by NCAR's Computational and Information Systems Laboratory, sponsored by the National Science Foundation. MB acknowledges support from the National Science Foundation Atmospheric and Geospace Sciences Postdoctoral Research Fellowship AGS-PRF-1524733, and from the Deutsche Forschungsgemeinschaft (DFG, German Research Foundation, project number 274762653, Collaborative Research Centre TRR 181 ("Energy Transfers in Atmosphere and Ocean").

\section{REFERENCES}

Banner, M. L., 1990: The influence of wave breaking on the surface pressure distribution in wind-wave interactions. J. Fluid Mech., 211, 463-495, https://doi.org/10.1017/S0022112090001653.

, and W. K. Melville, 1976: On the separation of air flow over water waves. J. Fluid Mech., 77, 825-842, https://doi.org/ 10.1017/S0022112076002905.
Black, P. G., and Coauthors, 2007: Air-sea exchange in hurricanes: Synthesis of Observations from the Coupled Boundary Layer Air-Sea Transfer Experiment. Bull. Amer. Meteor. Soc., 88, 357-374, https://doi.org/10.1175/BAMS-88-3-357.

Buckley, M. P., 2015: Structure of the airflow above surface waves. Ph.D. thesis, University of Delaware, 154 pp.

— , and F. Veron, 2016: Structure of the airflow above surface waves. J. Phys. Oceanogr., 46, 1377-1397, https://doi.org/ 10.1175/JPO-D-15-0135.1.

— and - 2017: Airflow measurements at a wavy air-water interface using PIV and LIF. Exp. Fluids, 58, 161, https:// doi.org/10.1007/s00348-017-2439-2.

— surface waves. Eur. J Fluid Mech., 73B, 132-143, https:// doi.org/10.1016/j.euromechflu.2018.04.003.

Chalikov, D., and S. Rainchik, 2011: Coupled numerical modelling of wind and waves and theory of the wave boundary layer. Bound.-Layer Meteor., 138, 1-41, https://doi.org/10.1007/ s10546-010-9543-7.

Donelan, M. A., B. K. Haus, N. Reul, W. J. Plant, M. Stiassnie, H. C. Graber, O. B. Brown, and E. S. Saltzman, 2004: On the limiting aerodynamic roughness of the ocean in very strong winds. Geophys. Res. Lett., 31, L18306, https://doi.org/10.1029/ 2004GL019460.

— , F. W. Dobson, S. D. Smith, and R. J. Anderson, 1993: On the dependence of sea surface roughness on wave development. J. Phys. Oceanogr., 23, 2143-2149, https://doi.org/10.1175/ 1520-0485(1993)023<2143:OTDOSS > 2.0.CO;2.

—, A. V. Babanin, I. R. Young, and M. L. Banner, 2006: Wavefollower field measurements of the wind-input spectral function. Part II: Parameterization of the wind input. J. Phys. Oceanogr., 36, 1672-1689, https://doi.org/10.1175/JPO2933.1.

_- M. Curcic, S. S. Chen, and A. K. Magnusson, 2012: Modeling waves and wind stress. J. Geophys. Res., 117, C00J23, https:// doi.org/10.1029/2011JC007787.

Edson, J. B., and Coauthors, 2013: On the exchange of momentum over the open ocean. J. Phys. Oceanogr., 43, 1589-1610, https://doi.org/10.1175/JPO-D-12-0173.1.

Fairall, C. W., J. D. Kepert, and G. J. Holland, 1994: The effect of sea spray on surface energy transports over the ocean. Global Atmos. Ocean Syst., 2, 121-142.

Gent, P. R., 1977: A numerical model of the air flow above water waves. Part 2. J. Fluid Mech., 82, 349-369, https://doi.org/ 10.1017/S0022112077000706.

_ and P. A. Taylor, 1976: A numerical model of the air flow above water waves. J. Fluid Mech., 77, 105-128, https://doi.org/ 10.1017/S0022112076001158.

Gong, W., P. A. Taylor, and A. Dörnbrack, 1996: Turbulent boundary-layer flow over fixed aerodynamically rough twodimensional sinusoidal waves. J. Fluid Mech., 312, 1-37, https://doi.org/10.1017/S0022112096001905.

Grare, L., W. L. Peirson, H. Branger, J. W. Walker, J.-P. Giovanangeli, and V. Makin, 2013: Growth and dissipation of wind-forced, deep-water waves. J. Fluid Mech., 722, 5-50, https://doi.org/10.1017/jfm.2013.88.

Hara, T., and S. E. Belcher, 2002: Wind forcing in the equilibrium range of wind-wave spectra. J. Fluid Mech., 470, 223-245, https://doi.org/10.1017/S0022112002001945.

_- and — 2004: Wind profile and drag coefficient over mature ocean surface wave spectra. J. Phys. Oceanogr., 34, 2345-2358, https://doi.org/10.1175/JPO2633.1.

_- , and P. Sullivan, 2015: Wave boundary layer turbulence over surface waves in a strongly forced condition. J. Phys. 
Oceanogr., 45, 868-883, https://doi.org/10.1175/JPO-D14-0116.1.

Holthuijsen, L. H., M. D. Powell, and J. D. Pietrzak, 2012: Wind and waves in extreme hurricanes. J. Geophys. Res., 117, C09003, https://doi.org/10.1029/2012JC007983.

Kudryavtsev, V. N., and V. K. Makin, 2001: The impact of air-flow separation on the drag of the sea surface. Bound-Layer Meteor., 98, 155-171, https://doi.org/10.1023/A:1018719917275.

— , and - 2002: Coupled dynamics of short waves and the airflow over long surface waves. J. Geophys. Res., 107, 3209, https://doi.org/10.1029/2001JC001251.

$\_, \ldots$, and J. F. Meirink, 2001: Simplified model of the air flow above waves. Bound.-Layer Meteor., 100, 63-90, https:// doi.org/10.1023/A:1018914113697.

Kukulka, T., and T. Hara, 2008: The effect of breaking waves on a coupled model of wind and ocean surface waves. Part II: Growing seas. J. Phys. Oceanogr., 38, 2164-2184, https:// doi.org/10.1175/2008JPO3962.1.

Makin, V. K., and V. N. Kudryavtsev, 1999: Coupled sea surfaceatmosphere model: 1 . Wind over waves coupling. J. Geophys. Res., 104, 7613-7623, https://doi.org/10.1029/1999JC900006.

Moeng, C.-H., 1984: A large-eddy-simulation model for the study of planetary boundary-layer turbulence. J. Atmos. Sci., 41, 2052-2062, https://doi.org/10.1175/1520-0469(1984)041<2052: ALESMF $>2.0 . \mathrm{CO} ; 2$.

- and P. P. Sullivan, 2015: Large eddy simulation. Encyclopedia of Atmospheric Sciences, 2nd ed. Academic Press, 232-240.

Moon, I.-J., I. Ginis, T. Hara, H. L. Tolman, C. W. Wright, and E. J. Walsh, 2003: Numerical simulation of sea surface directional wave spectra under hurricane wind forcing. J. Phys. Oceanogr., 33, 1680-1706, https://doi.org/10.1175/2410.1.

, T. Hara, I. Ginis, S. E. Belcher, and H. L. Tolman, 2004: Effect of surface waves on air-sea momentum exchange. Part I: Effect of mature and growing seas. J. Atmos. Sci., 61, 2321-2333, https://doi.org/10.1175/1520-0469(2004)061<2321: EOSWOA $>2.0 . \mathrm{CO} ; 2$.

Mueller, J. A., and F. Veron, 2009: Nonlinear formulation of the bulk surface stress over breaking waves: Feedback mechanisms from air-flow separation. Bound.-Layer Meteor., 130, 117-134, https://doi.org/10.1007/s10546-008-9334-6.

Oppenheim, A. V., and R. W. Schafer, 2013: Discrete-Time Signal Processing. Pearson Education, 1144 pp.

Peirson, W. L., and A. W. Garcia, 2008: On the wind-induced growth of slow water waves of finite steepness. J. Fluid Mech., 608, 243-274, https://doi.org/10.1017/S002211200800205X.

Powell, M. D., P. J. Vickery, and T. A. Reinhold, 2003: Reduced drag coefficient for high wind speeds in tropical cyclones. Nature, 422, 279-283, https://doi.org/10.1038/nature01481.
Reichl, B. G., T. Hara, and I. Ginis, 2014: Sea state dependence of the wind stress over the ocean under hurricane winds: Sea state dependence of the wind stress. J. Geophys. Res. Oceans, 119, 30-51, https://doi.org/10.1002/2013JC009289.

Reul, N., H. Branger, and J.-P. Giovanangeli, 2008: Air flow structure over short-gravity breaking water waves. Bound.-Layer Meteor., 126, 477-505, https://doi.org/10.1007/s10546-007-9240-3.

Richter, D., and P. Sullivan, 2013: Sea surface drag and the role of spray. Geophys. Res. Lett., 40, 656-660, https://doi.org/10.1002/ grl.50163.

Sullivan, P. P., J. C. McWilliams, and C.-H. Moeng, 2000: Simulation of turbulent flow over idealized water waves. J. Fluid Mech., 404, 47-85, https://doi.org/10.1017/S0022112099006965.

and E. G. Patton, 2014: Large-eddy simulation of marine atmospheric boundary layers above a spectrum of moving waves. J. Atmos. Sci., 71, 4001-4027, https://doi.org/10.1175/ JAS-D-14-0095.1.

—, M. L. Banner, R. P. Morison, and W. L. Peirson, 2018: Turbulent flow over steep steady and unsteady waves under strong wind forcing. J. Phys. Oceanogr., 48, 3-27, https://doi.org/ 10.1175/JPO-D-17-0118.1.

Taylor, P. K., and M. J. Yelland, 2001: The dependence of sea surface roughness on the height and steepness of the waves. J. Phys. Oceanogr., 31, 572-590, https://doi.org/10.1175/15200485(2001)031<0572:TDOSSR > 2.0.CO;2.

Teixeira, M., 2018: A model for the wind-driven current in the wavy oceanic surface layer: Apparent friction velocity reduction and roughness length enhancement. J. Phys. Oceanogr., 48, 2721-2736, https://doi.org/10.1175/JPO-D-18-0086.1.

Troitskaya, Y., D. Sergeev, O. Ermakova, and G. Balandina, 2011: Statistical parameters of the air turbulent boundary layer over steep water waves measured by the PIV technique. J. Phys. Oceanogr., 41, 1421-1454, https://doi.org/10.1175/2011JPO4392.1.

Uz, M., M. Donelan, T. Hara, and E. Bock, 2002: Laboratory studies of wind stress over surface waves. Bound.-Layer Meteor., 102, 301-331, https://doi.org/10.1023/A:1013119313063.

Veron, F., 2015: Ocean spray. Annu. Rev. Fluid Mech., 47, 507-538, https://doi.org/10.1146/annurev-fluid-010814-014651.

__, G. Saxena, and S. K. Misra, 2007: Measurements of the viscous tangential stress in the airflow above wind waves. Geophys. Res. Lett., 34, L19603, https://doi.org/10.1029/ 2007GL031242.

Yang, D., and L. Shen, 2010: Direct-simulation-based study of turbulent flow over various waving boundaries. J. Fluid Mech., 650, 131-180, https://doi.org/10.1017/S0022112009993557.

Zavadsky, A., and L. Shemer, 2012: Characterization of turbulent airflow over evolving water-waves in a wind-wave tank. J. Geophys. Res., 117, C00J19, https://doi.org/10.1029/2011JC007790. 